\title{
Hecke-Clifford Algebras and Spin Hecke Algebras IV: Odd Double Affine Type
}

Ta KHONGSAP and Weiqiang WANG

Department of Mathematics, University of Virginia, Charlottesville, VA 22904, USA

E-mail: tk7p@virginia.edu,ww9c@virginia.edu

URL: http://people.virginia.edu/ tk7p/, http://www.math.virginia.edu/ $\sim_{\text {ww9c/ }}$

Received October 15, 2008, in final form January 22, 2009; Published online January 28, 2009

doi:10.3842/SIGMA.2009.012

\begin{abstract}
We introduce an odd double affine Hecke algebra (DaHa) generated by a classical Weyl group $W$ and two skew-polynomial subalgebras of anticommuting generators. This algebra is shown to be Morita equivalent to another new DaHa which are generated by $W$ and two polynomial-Clifford subalgebras. There is yet a third algebra containing a spin Weyl group algebra which is Morita (super)equivalent to the above two algebras. We establish the PBW properties and construct Verma-type representations via Dunkl operators for these algebras.
\end{abstract}

Key words: spin Hecke algebras; Hecke-Clifford algebras; Dunkl operators

2000 Mathematics Subject Classification: 20C08

\section{Introduction}

1.1. The Dunkl operator [3], which is an ingenious mixture of differential and reflection operators, has found numerous applications to orthogonal polynomials, representation theory, noncommutative geometry, and so on in the past twenty years. To a large extent, the Dunkl operators helped to motivate the definition of double affine Hecke algebras of Cherednik, which have played important roles in several areas of mathematics. In recent years, the representation theory of a degenerate version of the double affine Hecke algebra (known as the rational Cherednik algebra or Cherednik-Dunkl algebra) has been studied extensively ([5, 4]; see the review paper of Rouquier [14] for extensive references).

In [16], the second author initiated a program of constructing the so-called spin Hecke algebras associated to Weyl groups with nontrivial 2-cocycles, by introducing the spin affine Hecke algebra as well as the rational and trignometric double affine Hecke algebras associated to the spin symmetric group of I. Schur [15]. Subsequently, in a series of papers [8, 9, 10, 17], the authors have extended the constructions of [16] in several different directions.

The construction of $[16,10]$ provided two (super)algebras $\ddot{\mathfrak{H}}_{W}^{\mathfrak{c}}$ and $\ddot{\mathfrak{H}}_{W}^{-}$associated to any classical Weyl group $W$ which are Morita super-equivalent in the sense of [17]. These algebras admit the following PBW type properties:

$$
\ddot{\mathfrak{H}}_{W}^{\mathfrak{c}} \cong \mathbb{C}\left[\mathfrak{h}^{*}\right] \otimes \mathcal{C}_{\mathfrak{h}^{*}} \otimes \mathbb{C} W \otimes \mathbb{C}[\mathfrak{h}], \quad \ddot{\mathfrak{H}}_{W}^{-} \cong \mathcal{C}\left\{\mathfrak{h}^{*}\right\} \otimes \mathbb{C} W^{-} \otimes \mathbb{C}[\mathfrak{h}] .
$$

Here we denote by $\mathfrak{h}$ the reflection representation of $W$, by $\mathbb{C}\left[\mathfrak{h}^{*}\right]$ the polynomial algebra on $\mathfrak{h}^{*}$, by $\mathcal{C}_{\mathfrak{h}^{*}}$ a Clifford algebra, by $\mathcal{C}\left\{\mathfrak{h}^{*}\right\}$ a skew-polynomial algebra with anti-commuting generators, and by $\mathbb{C} W^{-}$the spin Weyl group algebra associated to the element -1 in the Schur multiplier $H^{2}\left(W, \mathbb{C}^{*}\right)$.

\footnotetext{
${ }^{\star}$ This paper is a contribution to the Special Issue on Dunkl Operators and Related Topics. The full collection is available at http://www.emis.de/journals/SIGMA/Dunkl_operators.html
} 
In contrast to the rational Cherednik algebra (cf. $[5,14]$ ) which admits a nontrivial automorphism group, the construction of the algebras $\ddot{\mathfrak{H}}_{W}^{\mathfrak{c}}$ is asymmetric as $\ddot{\mathfrak{H}}_{W}^{\mathfrak{c}}$ contains as subalgebras one polynomial algebra and one polynomial-Clifford subalgebras $\mathbb{C}\left[\mathfrak{h}^{*}\right] \otimes \mathcal{C}_{\mathfrak{h}^{*}}$ (the polynomialClifford algebra also appeared in the affine Hecke-Clifford algebra of type $A$ introduced by Nazarov [13]). Moreover, in type $A$ case, $\ddot{\mathfrak{H}}_{W}^{\mathfrak{c}}$ contains Nazarov's algebra as a subalgebra, see $[16]$.

1.2. In the present paper, we introduce three new algebras $\mathbb{H}_{W}^{c c}, \mathbb{H}_{W}^{-c}$ and $\mathbb{H}_{W}$, which are shown to be Morita (super)equivalent to each other and to have PBW properties as follows:

$$
\begin{aligned}
& \mathbb{H}_{W}^{c c} \cong \mathbb{C}[\mathfrak{h}] \otimes \mathcal{C}_{\mathfrak{h}} \otimes \mathbb{C} W \otimes \mathcal{C}_{\mathfrak{h}^{*}} \otimes \mathbb{C}\left[\mathfrak{h}^{*}\right] \\
& \mathbb{H}_{W}^{-c} \cong \mathcal{C}\{\mathfrak{h}\} \otimes \mathbb{C} W^{-} \otimes \mathcal{C}_{\mathfrak{h}^{*}} \otimes \mathbb{C}\left[\mathfrak{h}^{*}\right] \\
& \mathbb{H}_{W} \cong \mathcal{C}\{\mathfrak{h}\} \otimes \mathbb{C} W \otimes \mathcal{C}\left\{\mathfrak{h}^{*}\right\} .
\end{aligned}
$$

A novel feature here is that the algebra $\mathbb{H}_{W}^{c c}$ contains two isomorphic copies of the polynomialClifford subalgebra and there is an automorphism of $\mathbb{H}_{W}^{c c}$ which switches these two copies. Similar remark applies to the algebra $\mathbb{H}_{W}$. We further show that the odd DaHa $\mathbb{H}_{W}$ of type $A$ contains the degenerate affine algebra of Drinfeld and Lusztig as a subalgebra (see [5] for a similar phenomenon).

It turns out that the number of parameters in the algebras $\mathbb{H}_{W}^{c c}, \mathbb{H}_{W}^{-c}$ and $\mathbb{H}_{W}$ is equal to one plus the number of conjugacy classes of reflections in $W$, which is the same as for the corresponding rational Cherednik algebras and differs by one from the algebras introduced in [16, 10]. However, in contrast to the usual Cherednik algebras, we show that each of the algebras $\mathbb{H}_{W}^{c c}$, $\mathbb{H}_{W}^{-c}$ and $\mathbb{H}_{W}$ contain large centers and are indeed module-finite over their respective centers.

1.3. In Section 2 we present a finite dimensional version of the Morita (super) equivalence of the DaHa mentioned above, and introduce the necessary concepts such as spin Weyl group algebras and Clifford algebras associated to the reflection representation $\mathfrak{h}$.

The Schur multipliers $H^{2}\left(W, \mathbb{C}^{*}\right)$ for finite Weyl groups $W$ were computed by Ihara and Yokonuma [6] (cf. Karpilovsky [7, Theorem 7.2.2]). For example, $H^{2}\left(W_{B_{n}}, \mathbb{C}^{*}\right)=\mathbb{Z}_{2} \times \mathbb{Z}_{2} \times \mathbb{Z}_{2}$ for $n \geq 4$. Given any finite Weyl group $W$ (not necessarily classical) and any 2-cocycle $\alpha \in$ $H^{2}\left(W, \mathbb{C}^{*}\right)$, we establish a superalgebra isomorphism (in two versions,+- )

$$
\dot{\Phi}_{ \pm}^{\alpha}: \mathcal{C}_{\mathfrak{h}^{*}} \rtimes_{ \pm} \mathbb{C} W^{-\alpha} \stackrel{\simeq}{\longrightarrow} \mathcal{C}_{\mathfrak{h}^{*}} \otimes \mathbb{C} W^{\alpha}
$$

For the purpose of the rest of this paper, only the case when $W$ is classical and $\alpha= \pm 1$ is needed. The special case when $\alpha=-1$ was established in [9], and this special case was in turn a generalization of a theorem of Sergeev and Yamaguchi for symmetric group.

We construct and study the algebras $\mathbb{H}_{W}^{c c}, \mathbb{H}_{W}^{-c}$ and $\mathbb{H}_{W}$ in the next three sections, i.e., in Sections 3, 4, and 5, respectively. Among other results, we establish the PBW properties as mentioned earlier and construct Verma-like representations of the three algebras via Dunkl operators. Note in particular that a representation for $\mathbb{H}_{W}$ (see Theorems 5.10, 5.13, and 5.14) is realized on the skew-polynomial algebra with anti-commuting Dunkl operators. Anti-commuting Dunkl operators first appeared in [16], also cf. [10]. In a very recent work [1], Bazlov and Berenstein introduced a notion of braided Cherednik algebra where anti-commuting Dunkl operators also make a natural appearance. After the second author communicated to them our construction of $\mathbb{H}_{W}$ for type $A$, they have also produced a similar algebra in their second version (cf. [1, Corollary 3.7]).

Finally, in the Appendix A, we collect the proofs of several lemmas stated in Section 3 and Section 5 . 


\section{Schur multipliers of Weyl groups and Clifford algebras}

\subsection{A distinguished double cover}

As in $[9,10]$, we shall be concerned about a distinguished double covering $\widetilde{W}$ of $W$ :

$$
1 \longrightarrow \mathbb{Z}_{2} \longrightarrow \widetilde{W} \longrightarrow W \longrightarrow 1 \text {. }
$$

We denote by $\mathbb{Z}_{2}=\{1, z\}$, and by $\tilde{t}_{i}$ a fixed preimage of the generators $s_{i}$ of $W$ for each $i$. The group $\widetilde{W}$ is generated by $z, \tilde{t}_{1}, \ldots, \tilde{t}_{n}$ with relations

$$
z^{2}=1, \quad\left(\tilde{t}_{i} \tilde{t}_{j}\right)^{m_{i j}}= \begin{cases}1, & \text { if } m_{i j}=1,3 \\ z, & \text { if } m_{i j}=2,4,6 .\end{cases}
$$

The quotient algebra $\mathbb{C} W^{-}:=\mathbb{C} \widetilde{W} /\langle z+1\rangle$ of $\mathbb{C} \widetilde{W}$ by the ideal generated by $z+1$ is called the spin Weyl group algebra associated to $W$. Denote by $t_{i} \in \mathbb{C} W^{-}$the image of $\tilde{t}_{i}$. It follows that $\mathbb{C} W^{-}$is isomorphic to the algebra generated by $t_{i}, 1 \leq i \leq n$, subject to the relations

$$
\left(t_{i} t_{j}\right)^{m_{i j}}=(-1)^{m_{i j}+1} \equiv\left\{\begin{aligned}
1, & \text { if } m_{i j}=1,3 \\
-1, & \text { if } m_{i j}=2,4,6 .
\end{aligned}\right.
$$

The algebra $\mathbb{C} W^{-}$has a natural superalgebra (i.e. $\mathbb{Z}_{2}$-graded) structure by letting each $t_{i}$ be odd.

Example 2.1. Let $W$ be the Weyl group of type $A_{n}, B_{n}$, or $D_{n}$, which will be considered extensively in later sections. Then the spin Weyl group algebra $\mathbb{C} W^{-}$is generated by $t_{1}, \ldots, t_{n}$ with relations listed in Table 2.1.

Table 2.1. The defining relations of $\mathbb{C} W^{-}$.

\begin{tabular}{|l|l|}
\hline Type of $W$ & Defining Relations for $\mathbb{C} W^{-}$ \\
\hline$A_{n}$ & $\begin{array}{l}t_{i}^{2}=1, t_{i} t_{i+1} t_{i}=t_{i+1} t_{i} t_{i+1}, \\
\left(t_{i} t_{j}\right)^{2}=-1 \text { if }|i-j|>1\end{array}$ \\
\hline \multirow{3}{*}{$B_{n}$} & $\begin{array}{l}t_{1}, \ldots, t_{n-1} \text { satisfy the relations for } \mathbb{C} W_{A_{n-1}}^{-} \\
t_{n}^{2}=1,\left(t_{i} t_{n}\right)^{2}=-1 \text { if } i \neq n-1, n, \\
\left(t_{n-1} t_{n}\right)^{4}=-1\end{array}$ \\
\hline$D_{n}$ & $\begin{array}{l}t_{1}, \ldots, t_{n-1} \text { satisfy the relations for } \mathbb{C} W_{A_{n-1}}^{-} \\
t_{n}^{2}=1,\left(t_{i} t_{n}\right)^{2}=-1 \text { if } i \neq n-2, n, \\
\\
t_{n-2} t_{n} t_{n-2}=t_{n} t_{n-2} t_{n}\end{array}$ \\
\hline
\end{tabular}

\subsection{Clifford algebra}

Denote by $\mathfrak{h}$ the reflection representation of the Weyl group $W$ (i.e. a Cartan subalgebra of the corresponding complex Lie algebra $\mathfrak{g}$ ). In the case of type $A_{n-1}$, we will always choose to work with the Cartan subalgebra $\mathfrak{h}=\mathbb{C}^{n}$ of $g l_{n}$ instead of $s l_{n}$ in this paper.

Note that $\mathfrak{h}$ carries a $W$-invariant nondegenerate bilinear form $(-,-)$, which gives rise to an identification $\mathfrak{h}^{*} \cong \mathfrak{h}$ and also a bilinear form on $\mathfrak{h}^{*}$ which will be again denoted by $(-,-)$. We identify $\mathfrak{h}^{*}$ with a suitable subspace of $\mathbb{C}^{N}$ in a standard fashion (cf. e.g. [9, Table in 2.3]). Then describe the simple roots $\left\{\alpha_{i}\right\}$ for $\mathfrak{g}$ using a standard orthonormal basis $\left\{e_{i}\right\}$ of $\mathbb{C}^{N}$. It follows that $\left(\alpha_{i}, \alpha_{j}\right)=-2 \cos \left(\pi / m_{i j}\right)$. 
Denote by $\mathcal{C}_{\mathfrak{h}^{*}}$ the Clifford algebra associated to $\left(\mathfrak{h}^{*},(-,-)\right)$, which is regarded as a subalgebra of the Clifford algebra $\mathcal{C}_{N}$ associated to $\left(\mathbb{C}^{N},(-,-)\right)$. We shall denote by $c_{i}$ the generator in $\mathcal{C}_{N}$ corresponding to $\sqrt{2} \mathfrak{e}_{i}$ and denote by $\beta_{i}$ the generator of $\mathcal{C}_{\mathfrak{h}^{*}}$ corresponding to the simple root $\alpha_{i}$ normalized with $\beta_{i}^{2}=1$. In particular, $\mathcal{C}_{N}$ is generated by $c_{1}, \ldots, c_{N}$ subject to the relations

$$
c_{i}^{2}=1, \quad c_{i} c_{j}=-c_{j} c_{i} \quad \text { if } \quad i \neq j .
$$

For example, we have

$$
\beta_{i}=\frac{1}{\sqrt{2}}\left(c_{i}-c_{i+1}\right), \quad 1 \leq i \leq n-1
$$

and an additional one

$$
\beta_{n}= \begin{cases}c_{n} & \text { if } W=W_{B_{n}}, \\ \frac{1}{\sqrt{2}}\left(c_{n-1}+c_{n}\right) & \text { if } W=W_{D_{n}} .\end{cases}
$$

Note that $N=n$ in the above three cases. For a complete list of $\beta_{i}$ for each Weyl group $W$, we refer to [9, Section 2] for details.

The action of $W$ on $\mathfrak{h}$ and $\mathfrak{h}^{*}$ preserves the bilinear form $(-,-)$ and thus $W$ acts as automorphisms of the algebra $\mathcal{C}_{\mathfrak{h}^{*}}$. This gives rise to a semi-direct product $\mathcal{C}_{\mathfrak{h}^{*}} \rtimes \mathbb{C W}$. Moreover, the algebra $\mathcal{C}_{\mathfrak{h}^{*}} \rtimes \mathbb{C} W$ naturally inherits the superalgebra structure by letting elements in $W$ be even and each $\beta_{i}$ be odd.

\subsection{A superalgebra isomorphism}

We recall the following result of Morris (the type $A$ case goes back to Schur).

Proposition $2.2([12,15])$. Let $W$ be a finite Weyl group. Then, there exists a surjective superalgebra homomorphism $\Omega: \mathbb{C} W^{-} \longrightarrow \mathcal{C}_{\mathfrak{h}^{*}}$ which sends $t_{i}$ to $\beta_{i}$ for each $i$.

Given two superalgebras $\mathcal{A}$ and $\mathcal{B}$, we view the tensor product of superalgebras $\mathcal{A} \otimes \mathcal{B}$ as a superalgebra with multiplication defined by

$$
(a \otimes b)\left(a^{\prime} \otimes b^{\prime}\right)=(-1)^{|b|\left|a^{\prime}\right|}\left(a a^{\prime} \otimes b b^{\prime}\right) \quad\left(a, a^{\prime} \in \mathcal{A}, b, b^{\prime} \in \mathcal{B}\right),
$$

where $|b|$ denotes the $\mathbb{Z}_{2}$-degree of $b$, etc.

Now, let $\mathcal{C}_{n} \rtimes_{-} \mathbb{C} W^{-}$denote the algebra generated by the subalgebras $\mathcal{C}_{n}$ and $\mathbb{C} W^{-}$with the following additional multiplication:

$$
t_{i} c_{j}=-c_{j}^{s_{i}} t_{i} \quad \forall i, j .
$$

Note that $\mathcal{C}_{n} \rtimes_{-} \mathbb{C} W^{-}$has a natural superalgebra structure by setting each $c_{i}$ and $t_{j}$ to be odd for all admissible $i, j$. We also endow a superalgebra structure on $\mathcal{C}_{\mathfrak{h}^{*}} \otimes \mathbb{C} W$ by declaring all elements of $W$ to be even.

Theorem 2.3. We have an isomorphism of superalgebras:

$$
\dot{\Phi}: \mathcal{C}_{\mathfrak{h}^{*}} \rtimes_{-} \mathbb{C} W^{-} \stackrel{\simeq}{\longrightarrow} \mathcal{C}_{\mathfrak{h}^{*}} \otimes \mathbb{C} W
$$

which extends the identity map on $\mathcal{C}_{\mathfrak{h}^{*}}$ and sends each $t_{i}$ to $\beta_{i} s_{i}$. The inverse map $\dot{\Psi}$ is an extension of the identity map on $\mathcal{C}_{\mathfrak{h}^{*}}$ and sends each $s_{i}$ to $\beta_{i} t_{i}$.

We first prepare a few lemmas.

Lemma 2.4. We have $\left(\dot{\Phi}\left(t_{i}\right) \dot{\Phi}\left(t_{j}\right)\right)^{m_{i j}}=(-1)^{m_{i j}+1}$. 
Proof. Proposition 2.2 says that $\left(t_{i} t_{j}\right)^{m_{i j}}=\left(\beta_{i} \beta_{j}\right)^{m_{i j}}=(-1)^{m_{i j}+1}$. Also recall that $\left(s_{i} s_{j}\right)^{m_{i j}}=1$. Then we have

$$
\left(\dot{\Phi}\left(t_{i}\right) \dot{\Phi}\left(t_{j}\right)\right)^{m_{i j}}=\left(\beta_{i} s_{i} \beta_{j} s_{j}\right)^{m_{i j}}=\left(\beta_{i} \beta_{j}\right)^{m_{i j}}\left(s_{i} s_{j}\right)^{m_{i j}}=(-1)^{m_{i j}+1} .
$$

Lemma 2.5. We have $\beta_{j} \dot{\Phi}\left(t_{i}\right)=-\dot{\Phi}\left(t_{i}\right) \beta_{j}^{s_{i}}$ for all $i, j$.

Proof. Note that $\left(\beta_{i}, \beta_{i}\right)=2 \beta_{i}^{2}=2$, and hence

$$
\beta_{j} \beta_{i}=-\beta_{i} \beta_{j}+\left(\beta_{j}, \beta_{i}\right)=-\beta_{i} \beta_{j}+\frac{2\left(\beta_{j}, \beta_{i}\right)}{\left(\beta_{i}, \beta_{i}\right)} \beta_{i}^{2}=-\beta_{i} \beta_{j}^{s_{i}} .
$$

Thus, we have

$$
\beta_{j} \dot{\Phi}\left(t_{i}\right)=\beta_{j} \beta_{i} s_{i}=-\beta_{i} \beta_{j}^{s_{i}} s_{i}=-\beta_{i} s_{i} \beta_{j}^{s_{i}}=-\dot{\Phi}\left(t_{i}\right) \beta_{j}^{s_{i}} .
$$

Proof of Theorem 2.3. The algebra $\mathcal{C}_{\mathfrak{h}^{*}} \rtimes_{-} \mathbb{C} W^{-}$is generated by $\beta_{i}$ and $t_{i}$ for all $i$. Lemmas 2.4 and 2.5 imply that $\dot{\Phi}$ is a (super) algebra homomorphism. Clearly $\dot{\Phi}$ is surjective, and thus an isomorphism by a dimension counting argument.

Clearly, $\dot{\Psi}$ and $\dot{\Phi}$ are inverses of each other.

Let us denote by $\mathcal{C}_{\mathfrak{h}^{*} \oplus \mathfrak{h}}$ the Clifford algebra associated to $\left(\left(\mathfrak{h}^{*},(-,-)\right) \oplus(\mathfrak{h},(-,-))\right)$, and regard it as a subalgebra of the Clifford algebra $\mathcal{C}_{2 N}$ associated to $\left(\left(\mathbb{C}^{N},(-,-)\right) \oplus\left(\left(\mathbb{C}^{N}\right)^{*},(-,-)\right)\right)$. We shall denote by $e_{i}$ and $\nu_{i}$ the counterparts to $c_{i}$ and $\beta_{i}$ via the identification $\mathcal{C}_{\mathfrak{h}^{*}} \cong \mathcal{C}_{\mathfrak{h}}$.

By [9, Theorem 2.4], there exists an isomorphism of superalgebras

$$
\Phi: \mathcal{C}_{\mathfrak{h}} \rtimes \mathbb{C} W \rightarrow \mathcal{C}_{\mathfrak{h}} \otimes \mathbb{C} W^{-}
$$

which extends the identity map on $\mathcal{C}_{\mathfrak{h}}$ and sends each $s_{i}$ to $-\sqrt{-1} \nu_{i} t_{i}$. The isomorphism $\Phi$ was due to Sergeev and Yamaguchi when $W$ is the symmetric group.

Theorem 2.6. We have an isomorphism of superalgebras:

$$
\ddot{\Phi}: \mathcal{C}_{\mathfrak{h}^{*} \oplus \mathfrak{h}} \rtimes \mathbb{C} W \stackrel{\simeq}{\longrightarrow} \mathcal{C}_{\mathfrak{h}^{*} \oplus \mathfrak{h}} \otimes \mathbb{C} W
$$

which extends the identity map on $\mathcal{C}_{\mathfrak{h}^{*} \oplus \mathfrak{h}}$ and sends each $s_{i}$ to $\sqrt{-1} \beta_{i} \nu_{i} s_{i}$. The inverse map $\ddot{\Psi}$ is the extension of the identity map on $\mathcal{C}_{\mathfrak{h}^{*} \oplus \mathfrak{h}}$ which sends each $s_{i}$ to $\sqrt{-1} \beta_{i} \nu_{i} s_{i}$.

Proof. The isomorphisms $\dot{\Phi}$ in Theorem 2.3 and $\Phi$ in (2.1) can be readily extended to the following isomorphisms of superalgebras which restrict to the identity map on $\mathcal{C}_{\mathfrak{h}^{*} \oplus \mathfrak{h}}$ :

$$
\begin{aligned}
& \Phi: \mathcal{C}_{\mathfrak{h}^{*} \oplus \mathfrak{h}} \rtimes \mathbb{C} W \stackrel{\simeq}{\longrightarrow} \mathcal{C}_{\mathfrak{h}} \otimes\left(\mathcal{C}_{\mathfrak{h}^{*}} \rtimes_{-} \mathbb{C} W^{-}\right), \\
& \dot{\Phi}: \mathcal{C}_{\mathfrak{h}} \otimes\left(\mathcal{C}_{\mathfrak{h}^{*}} \rtimes_{-} \mathbb{C} W^{-}\right) \stackrel{\simeq}{\longrightarrow} \mathcal{C}_{\mathfrak{h}^{*} \oplus \mathfrak{h}} \otimes \mathbb{C} W .
\end{aligned}
$$

Observe that $\ddot{\Phi}=\dot{\Phi} \circ \Phi$, and so $\ddot{\Phi}$ is an isomorphism.

\subsection{The case of general 2-cocycles}

The materials of this subsection generalize the Section 2.3 above and [9, Section 2]; however, they will not be used in subsequent sections.

The Schur multipliers $H^{2}\left(W, \mathbb{C}^{*}\right)$ for finite Weyl groups $W$ were computed by Ihara and Yokonuma [6] (also cf. Karpilovsky [7, Theorem 7.2.2]). In all cases, we have $H^{2}\left(W, \mathbb{C}^{*}\right) \cong \prod_{j=1}^{k} \mathbb{Z}_{2}$ for suitable $k=0,1,2,3$. 
Consider the following central extension of $W$ by $H^{2}\left(W, \mathbb{C}^{*}\right)$ :

$$
1 \longrightarrow H^{2}\left(W, \mathbb{C}^{*}\right) \longrightarrow \widetilde{W} \longrightarrow W \longrightarrow 1 \text {. }
$$

We denote by $z_{i}$ the generator of the $i$ th copy of $\mathbb{Z}_{2}$ in $H^{2}\left(W, \mathbb{C}^{*}\right) \cong \prod_{j=1}^{k} \mathbb{Z}_{2}$ and by $t_{i}$ a fixed preimage of the generator $s_{i}$ of $\mathrm{W}$ for each $i$. The group $\widetilde{W}$ is generated by $z_{1}, \ldots, z_{k}, t_{1}, \ldots, t_{n}$ subject to that $z_{i}$ is central of order 2 for all $i$, and the additional relations shown in Table 2.2 below (cf. [7, Table 7.1]). In particular, the values of $k$ can be read off from Table 2.2.

Table 2.2. Central extensions $\widetilde{W}$ of Weyl groups.

\begin{tabular}{|c|c|}
\hline Type of $W$ & Generators/Relations for $\widetilde{W}$ \\
\hline$A_{n}(n \geq 3)$ & $\begin{array}{l}t_{i}^{2}=1,1 \leq i \leq n \\
t_{i} t_{i+1} t_{i}=t_{i+1} t_{i} t_{i+1}, 1 \leq i \leq n-1 \\
t_{i} t_{j}=z_{1} t_{j} t_{i} \text { if } m_{i j}=2\end{array}$ \\
\hline$B_{2}$ & $t_{1}^{2}=t_{2}^{2}=1,\left(t_{1} t_{2}\right)^{2}=z_{1}\left(t_{2} t_{1}\right)^{2}$ \\
\hline$B_{3}$ & $\begin{array}{l}t_{1}^{2}=t_{2}^{2}=t_{3}^{2}=1, t_{1} t_{2} t_{1}=t_{2} t_{1} t_{2} \\
t_{1} t_{3}=z_{1} t_{3} t_{1},\left(t_{2} t_{3}\right)^{2}=z_{2}\left(t_{3} t_{2}\right)^{2}\end{array}$ \\
\hline$B_{n}(n \geq 4)$ & $\begin{array}{l}t_{i}^{2}=1,1 \leq i \leq n, t_{i} t_{i+1} t_{i}=t_{i+1} t_{i} t_{i+1}, 1 \leq i \leq n-2 \\
t_{i} t_{j}=z_{1} t_{j} t_{i}, 1 \leq i<j \leq n-1, m_{i j}=2 \\
t_{i} t_{n}=z_{2} t_{n} t_{i}, 1 \leq i \leq n-2 \\
\left(t_{n-1} t_{n}\right)^{2}=z_{3}\left(t_{n} t_{n-1}\right)^{2}\end{array}$ \\
\hline$D_{4}$ & $\begin{array}{l}t_{i}^{2}=1,1 \leq i \leq 4, t_{i} t_{j} t_{i}=t_{j} t_{i} t_{j} \text { if } m_{i j}=3 \\
t_{1} t_{3}=z_{1} t_{3} t_{1}, t_{1} t_{4}=z_{2} t_{4} t_{1}, t_{3} t_{4}=z_{3} t_{4} t_{3}\end{array}$ \\
\hline$D_{n}(n \geq 5)$ & $\begin{array}{l}t_{i}^{2}=1,1 \leq i \leq n, t_{i} t_{j} t_{i}=t_{j} t_{i} t_{j} \text { if } m_{i j}=3 \\
t_{i} t_{j}=z_{1} t_{j} t_{i}, 1 \leq i<j \leq n, m_{i j}=2, i \neq n-1 \\
t_{n-1} t_{n}=z_{2} t_{n} t_{n-1}\end{array}$ \\
\hline$E_{n=6,7,8}$ & $\begin{array}{l}t_{i}^{2}=1,1 \leq i \leq n, t_{i} t_{j} t_{i}=t_{j} t_{i} t_{j} \text { if } m_{i j}=3 \\
t_{i} t_{j}=z_{1} t_{j} t_{i}, \text { if } m_{i j}=2\end{array}$ \\
\hline$F_{4}$ & $\begin{array}{l}t_{i}^{2}=1,1 \leq i \leq 4, t_{i} t_{i+1} t_{i}=t_{i+1} t_{i} t_{i+1}(i=1,3) \\
t_{i} t_{j}=z_{1} t_{j} t_{i}, 1 \leq i<j \leq 4, m_{i j}=2 \\
\left(t_{2} t_{3}\right)^{2}=z_{2}\left(t_{3} t_{2}\right)^{2}\end{array}$ \\
\hline$G_{2}$ & $t_{1}^{2}=t_{2}^{2}=1,\left(t_{1} t_{2}\right)^{3}=z_{1}\left(t_{2} t_{1}\right)^{3}$ \\
\hline
\end{tabular}

For $\alpha=\left(\alpha_{i}\right)_{i=1, \ldots, k} \in H^{2}\left(W, \mathbb{C}^{*}\right)$, the quotient $\mathbb{C} W^{\alpha}:=\mathbb{C} \widetilde{W} /\left\langle z_{i}-\alpha_{i}, \forall i\right\rangle$ can be identified as the algebra generated by $t_{1}, \ldots, t_{n}$ subject to the relations:

$$
\left(t_{i} t_{j}\right)^{m_{i j}}=\left\{\begin{aligned}
1, & \text { if } m_{i j}=1,3 \\
\alpha_{i j}, & \text { if } m_{i j}=2,4,6
\end{aligned}\right.
$$

where $\alpha_{i j} \in\{ \pm 1\}$ is specified by $\alpha \in H^{2}\left(W, \mathbb{C}^{*}\right)$ as in Table 2.2 .

Let $\mathcal{C}_{\mathfrak{h}^{*}} \rtimes_{-} \mathbb{C} W^{-\alpha}$ denote the algebra generated by subalgebras $\mathcal{C}_{\mathfrak{h}^{*}}$ and $\mathbb{C} W^{-\alpha}$ with the following additional multiplication:

$$
t_{i}^{-} \beta_{j}=-\beta_{j}^{s_{i}} t_{i}^{-} \quad \forall i, j
$$


where we have denoted by $t_{i}^{-}$the generators of the subalgebra $\mathbb{C} W^{-\alpha}$ of $\mathcal{C}_{\mathfrak{h}^{*}} \rtimes_{-} \mathbb{C} W^{-\alpha}$, in order to distinguish from the generators $t_{i}$ of $\mathbb{C} W^{\alpha}$ below. We impose superalgebra structures on the algebras $\mathcal{C}_{\mathfrak{h}^{*}} \rtimes_{-} \mathbb{C} W^{-\alpha}$ and on $\mathcal{C}_{\mathfrak{h}^{*}} \otimes \mathbb{C} W^{\alpha}$ by letting $t_{i}^{-}$be odd, $t_{i}$ be even, and $\beta_{i}$ be odd for all $i$.

Theorem 2.7. Fix a 2-cocycle $\alpha \in H^{2}\left(W, \mathbb{C}^{*}\right)$. We have an isomorphism of superalgebras:

$$
\dot{\Phi}_{-}^{\alpha}: \mathcal{C}_{\mathfrak{h}^{*}} \rtimes_{-} \mathbb{C} W^{-\alpha} \stackrel{\simeq}{\longrightarrow} \mathcal{C}_{\mathfrak{h}^{*}} \otimes \mathbb{C} W^{\alpha}
$$

which extends the identity map on $\mathcal{C}_{\mathfrak{h}^{*}}$ and sends $t_{i}^{-}$to $\beta_{i} t_{i}$ for each $i$. The inverse map $\dot{\Psi}_{-}^{\alpha}$ is the extension of the identity map on $\mathcal{C}_{\mathfrak{h}^{*}}$ and sends $t_{i}$ to $\beta_{i} t_{i}^{-}$for each $i$.

Proof. By Lemma 2.5, we have $\beta_{j} \beta_{i}=-\beta_{i} \beta_{j}^{s_{i}}$. Recall that $t_{i}^{-}$is odd and $t_{i}$ is even. So we have $\beta_{j} \dot{\Phi}_{-}^{\alpha}\left(t_{i}^{-}\right)=-\dot{\Phi}_{-}^{\alpha}\left(t_{i}^{-}\right) \beta_{j}^{s_{i}}$ for all admissible $i, j$. Moreover,

$$
\begin{aligned}
\left(\dot{\Phi}_{-}^{\alpha}\left(t_{i}^{-}\right) \dot{\Phi}_{-}^{\alpha}\left(t_{j}^{-}\right)\right)^{m_{i j}} & =\left(\beta_{i} t_{i} \beta_{j} t_{j}\right)^{m_{i j}}=\left(\beta_{i} \beta_{j}\right)^{m_{i j}}\left(t_{i} t_{j}\right)^{m_{i j}}=(-1)^{m_{i j}+1}\left(t_{i} t_{j}\right)^{m_{i j}} \\
& = \begin{cases}1 & \text { if } m_{i j}=1,3, \\
-\alpha_{i j} & \text { if } m_{i j}=2,4,6 .\end{cases}
\end{aligned}
$$

Clearly, $\dot{\Phi}_{-}^{\alpha}$ preserves the $\mathbb{Z}_{2}$-grading. Hence, it follows that $\dot{\Phi}_{-}^{\alpha}$ is a surjective superalgebra homomorphism, and thus an isomorphism by dimension counting. It is clear that $\dot{\Psi}_{-}^{\alpha}$ is the inverse of $\dot{\Phi}_{-}^{\alpha}$.

Denote by $\mathcal{C}_{\mathfrak{h}^{*}} \rtimes_{+} \mathbb{C} W^{-\alpha}$ the algebra generated by subalgebras $\mathcal{C}_{\mathfrak{h}^{*}}$ and $\mathbb{C} W^{-\alpha}$ with the following additional multiplication:

$$
t_{i}^{+} \beta_{j}=\beta_{j}^{s_{i}} t_{i}^{+} \quad \forall i, j,
$$

where we have denoted by $t_{i}^{+}$the generators of the subalgebra $\mathbb{C} W^{-\alpha}$ of $\mathcal{C}_{\mathfrak{h}^{*}} \rtimes_{+} \mathbb{C} W^{-\alpha}$, in order to distinguish from the generators $t_{i}$ of $\mathbb{C} W^{\alpha}$. We impose superalgebra structures on the algebras $\mathcal{C}_{\mathfrak{h}^{*}} \rtimes_{+} \mathbb{C} W^{-\alpha}$ and on $\mathcal{C}_{\mathfrak{h}^{*}} \otimes \mathbb{C} W^{\alpha}$ by letting $t_{i}^{+}$be even, $t_{i}$ be odd, and $\beta_{i}$ be odd for all $i$.

Theorem 2.8. Fix a 2-cocycle $\alpha \in H^{2}\left(W, \mathbb{C}^{*}\right)$. We have an isomorphism of superalgebras:

$$
\dot{\Phi}_{+}^{\alpha}: \mathcal{C}_{\mathfrak{h}^{*}} \rtimes_{+} \mathbb{C} W^{-\alpha} \stackrel{\simeq}{\longrightarrow} \mathcal{C}_{\mathfrak{h}^{*}} \otimes \mathbb{C} W^{\alpha}
$$

which extends the identity map on $\mathcal{C}_{\mathfrak{h}^{*}}$ and sends $t_{i}^{+} \mapsto-\sqrt{-1} \beta_{i} t_{i}$. The inverse map $\dot{\Psi}_{+}^{\alpha}$ is the extension of the identity map on $\mathcal{C}_{\mathfrak{h}^{*}}$ and sends each $t_{i}$ to $\sqrt{-1} \beta_{i} t_{i}^{+}$.

Proof. By Lemma 2.5, we have $\beta_{j} \beta_{i}=-\beta_{i} \beta_{j}^{s_{i}}$. Recall that $t_{i}^{+}$is even while $t_{i}$ is odd. Then $\beta_{j} \dot{\Phi}_{+}^{\alpha}\left(t_{i}^{+}\right)=\dot{\Phi}_{+}^{\alpha}\left(t_{i}^{+}\right) \beta_{j}^{s_{i}}$ for all admissible $i, j$. Moreover,

$$
\begin{aligned}
\left(\dot{\Phi}_{+}^{\alpha}\left(t_{i}^{+}\right) \dot{\Phi}_{+}^{\alpha}\left(t_{j}^{+}\right)\right)^{m_{i j}} & =\left(-\beta_{i} t_{i} \beta_{j} t_{j}\right)^{m_{i j}}=\left(\beta_{i} \beta_{j} t_{i} t_{j}\right)^{m_{i j}}=\left(\beta_{i} \beta_{j}\right)^{m_{i j}}\left(t_{i} t_{j}\right)^{m_{i j}} \\
& = \begin{cases}1 & \text { if } m_{i j}=1,3, \\
-\alpha_{i j} & \text { if } m_{i j}=2,4,6 .\end{cases}
\end{aligned}
$$

It follows that $\dot{\Phi}_{+}^{\alpha}$ is an isomorphism of superalgebra with inverse $\dot{\Psi}_{+}^{\alpha}$.

Denote by $\mathcal{C}_{\mathfrak{h}^{*} \oplus \mathfrak{h}} \rtimes_{+} \mathbb{C} W^{\alpha}$ the algebra generated by subalgebras $\mathcal{C}_{\mathfrak{h}^{*} \oplus \mathfrak{h}}$ and $\mathbb{C} W^{\alpha}$ with the following additional multiplication:

$$
t_{i} \beta_{j}=\beta_{j}^{s_{i}} t_{i}, \quad t_{i} \nu_{j}=\nu_{j}^{s_{i}} t_{i}, \quad \forall i, j .
$$

We impose superalgebra structures on the algebras $\mathcal{C}_{\mathfrak{h}^{*} \oplus \mathfrak{h}} \rtimes_{+} \mathbb{C} W^{\alpha}$ and on $\mathcal{C}_{\mathfrak{h}^{*} \oplus \mathfrak{h}} \otimes \mathbb{C} W^{\alpha}$ by letting each $t_{i}$ be even, and letting each $\beta_{i}, \nu_{i}$ be odd. 
Corollary 2.9. For a 2-cocycle $\alpha \in H^{2}\left(W, \mathbb{C}^{*}\right)$, we have an isomorphism of superalgebras:

$$
\mathcal{C}_{\mathfrak{h}^{*} \oplus \mathfrak{h}} \rtimes_{+} \mathbb{C} W^{\alpha} \cong \mathcal{C}_{\mathfrak{h}^{*} \oplus \mathfrak{h}} \otimes \mathbb{C} W^{\alpha}
$$

which extends the identity map on $\mathcal{C}_{\mathfrak{h}^{*} \oplus \mathfrak{h}}$ and sends each $t_{i}$ to $\sqrt{-1} \beta_{i} \nu_{i} t_{i}$.

Remark 2.10. When $\alpha= \pm 1$ and $\mathbb{C} W^{\alpha}$ becomes the usual group algebra $\mathbb{C} W$ or the spin group algebra $\mathbb{C} W^{-}$, we recover the main results of Section 2.3.

\section{The DaHa with two polynomial-Clifford subalgebras}

In the remainder of the paper, $W$ is always assumed to be one of the classical Weyl groups of type $A_{n-1}, B_{n}$, or $D_{n}$, and we shall often write $\mathcal{C}_{2 n}$ for $\mathcal{C}_{\mathfrak{h}^{*} \oplus \mathfrak{h}}$.

\subsection{The definition of $\mathbb{H}_{W}^{c c}$}

Let $W$ be one of the classical Weyl groups. The goal of this section is to introduce a rational double affine Hecke algebra (DaHa) $\mathbb{H}_{W}^{c c}$ which is generated by $\mathbb{C} W$ and two isomorphic "polynomial-Clifford" subalgebras. Note that this construction is different from the double affine Hecke-Clifford algebra introduced in $[16,10]$ which is generated by $\mathbb{C} W$, a polynomial subalgebra, and a "polynomial-Clifford" subalgebra.

Identify $\mathbb{C}\left[\mathfrak{h}^{*}\right] \cong \mathbb{C}\left[x_{1}, \ldots, x_{n}\right]$ and $\mathbb{C}[\mathfrak{h}] \cong \mathbb{C}\left[y_{1}, \ldots, y_{n}\right]$, where $x_{i}, y_{i}(1 \leq i \leq n)$ correspond to the standard orthonormal basis $\left\{\mathfrak{e}_{i}\right\}$ for $\mathfrak{h}^{*}$ and its dual basis $\left\{\mathfrak{e}_{i}^{*}\right\}$ for $\mathfrak{h}$, respectively. For $x, y$ in an algebra $A$, we denote as usual that

$$
[x, y]=x y-y x \in A \text {. }
$$

\subsubsection{The algebra $\mathbb{H}_{W}^{c c}$ of type $A_{n-1}$}

Definition 3.1. Let $t, u \in \mathbb{C}$ and $W=S_{n}$. The algebra $\mathbb{H}_{W}^{c c}$ of type $A_{n-1}$ is generated by $x_{i}$, $y_{i}(1 \leq i \leq n), \mathcal{C}_{2 n}$ and $W$, subject to the following additional relations:

$$
\begin{aligned}
& x_{i} x_{j}=x_{j} x_{i}, \quad y_{i} y_{j}=y_{j} y_{i} \quad(\forall i, j), \\
& \sigma c_{i}=c_{i}^{\sigma} \sigma, \quad \sigma e_{i}=e_{i}^{\sigma} \sigma, \\
& \sigma x_{i}=x_{i}^{\sigma} \sigma, \quad \sigma y_{i}=y_{i}^{\sigma} \sigma \quad(\forall \sigma \in W, \forall i), \\
& e_{i} x_{j}=x_{j} e_{i}, \quad c_{i} x_{j}=(-1)^{\delta_{i j}} x_{j} c_{i} \quad(\forall i, j), \\
& c_{i} y_{j}=y_{j} c_{j}, \quad e_{i} y_{j}=(-1)^{\delta_{i j}} y_{j} e_{i} \quad(\forall i, j), \\
& {\left[y_{i}, x_{j}\right]=u\left(1+c_{i} c_{j}\right)\left(1+e_{j} e_{i}\right) s_{i j} \quad(i \neq j),} \\
& {\left[y_{i}, x_{i}\right]=t c_{i} e_{i}-u \sum_{k \neq i}\left(1+c_{k} c_{i}\right)\left(1+e_{k} e_{i}\right) s_{k i} .}
\end{aligned}
$$

Alternatively, we may view $t, u$ as formal variables and $\mathbb{H}_{W}^{c c}$ as a $\mathbb{C}[t, u]$-algebra. Similar remarks apply to other algebras defined in this paper.

\subsubsection{The algebra $\mathbb{H}_{W}^{c c}$ of type $D_{n}$}

Let $W=W_{D_{n}}$. Regarding elements in $W$ as even signed permutations of $1,2, \ldots, n$ as usual, we identify the generators $s_{i} \in W, 1 \leq i \leq n-1$, with transposition $(i, i+1)$, and $s_{n} \in W$ with the transposition of $(n-1, n)$ coupled with the sign changes at $n-1, n$. For $1 \leq i \neq j \leq n$, we denote by $s_{i j} \equiv(i, j) \in W$ the transposition of $i$ and $j$, and $\bar{s}_{i j} \equiv \overline{(i, j)} \in W$ the transposition of $i$ and $j$ coupled with the sign changes at $i, j$. By convention, we have

$$
\bar{s}_{n-1, n} \equiv \overline{(n-1, n)}=s_{n}, \quad \bar{s}_{i j} \equiv \overline{(i, j)}=s_{j n} s_{i, n-1} s_{n} s_{i, n-1} s_{j n} .
$$


Definition 3.2. Let $t, u \in \mathbb{C}$ and $W=W_{D_{n}}$. The algebra $\mathbb{H}_{W}^{c c}$ of type $D_{n}$ is generated by $x_{i}$, $y_{i}(1 \leq i \leq n), \mathcal{C}_{2 n}$ and $W$, subject to the relations (3.1) with the current $W$, and (3.3a)-(3.3b) with $i \neq j$ below:

$$
\begin{aligned}
& {\left[y_{i}, x_{j}\right]=u\left(1+c_{i} c_{j}\right)\left(1+e_{j} e_{i}\right) s_{i j}-u\left(1-c_{i} c_{j}\right)\left(1-e_{j} e_{i}\right) \bar{s}_{i j},} \\
& {\left[y_{i}, x_{i}\right]=t c_{i} e_{i}-u \sum_{k \neq i}\left(1+c_{k} c_{i}\right)\left(1+e_{k} e_{i}\right) s_{k i}+u \sum_{k \neq i}\left(1-c_{k} c_{i}\right)\left(1-e_{k} e_{i}\right) \bar{s}_{k i} .}
\end{aligned}
$$

\subsubsection{The algebra $\mathbb{H}_{W}^{c c}$ of type $B_{n}$}

Let $W=W_{B_{n}}$. We identify $W$ as usual with the signed permutations on $1, \ldots, n$. Regarding $W_{D_{n}}$ as a subgroup of $W$, we have $s_{i j}, \bar{s}_{i j} \in W$ for $1 \leq i \neq j \leq n$. Further denote $\tau_{i} \equiv \overline{(i)} \in W$ the sign change at $i$ for $1 \leq i \leq n$. By definition, we have

$$
\tau_{n} \equiv \overline{(n)}=s_{n}, \quad \tau_{i} \equiv \overline{(i)}=s_{i n} s_{n} s_{i n}
$$

Definition 3.3. Let $t, u, v \in \mathbb{C}$, and $W=W_{B_{n}}$. The algebra $\mathbb{H}_{W}^{c c}$ of type $B_{n}$ is generated by $x_{i}$, $y_{i}(1 \leq i \leq n), \mathcal{C}_{2 n}$ and $W$, subject to the relations (3.1) with the current $W$, and (3.4a) $-(3.4 \mathrm{~b})$ with $i \neq j$ below:

$$
\begin{aligned}
& {\left[y_{i}, x_{j}\right]=u\left(1+c_{i} c_{j}\right)\left(1+e_{j} e_{i}\right) s_{i j}-u\left(1-c_{i} c_{j}\right)\left(1-e_{j} e_{i}\right) \bar{s}_{i j},} \\
& {\left[y_{i}, x_{i}\right]=t c_{i} e_{i}-u \sum_{k \neq i}\left(1+c_{k} c_{i}\right)\left(1+e_{k} e_{i}\right) s_{k i}+u \sum_{k \neq i}\left(1-c_{k} c_{i}\right)\left(1-e_{k} e_{i}\right) \bar{s}_{k i}-v \tau_{i} .}
\end{aligned}
$$

\subsection{The PBW basis for $\mathbb{H}_{W}^{c c}$}

We shall denote $x^{\alpha}=x_{1}^{a_{1}} \cdots x_{n}^{a_{n}}$ for $\alpha=\left(a_{1}, \ldots, a_{n}\right) \in \mathbb{Z}_{+}^{n}, c^{\epsilon}=c_{1}^{\epsilon_{1}} \cdots c_{n}^{\epsilon_{n}}$ for $\epsilon=\left(\epsilon_{1}, \ldots, \epsilon_{n}\right) \in \mathbb{Z}_{2}^{n}$. Similarly, we define $y^{\alpha}$ and $e^{\epsilon}$. Note that the algebra $\mathbb{H}_{W}^{c c}$ contains $\mathbb{C}\left[\mathfrak{h}^{*}\right], \mathcal{C}_{\mathfrak{h}^{*}}, \mathbb{C}[\mathfrak{h}], \mathcal{C}_{\mathfrak{h}}$, and $\mathbb{C} W$ as subalgebras.

Theorem 3.4. Let $W$ be $W_{A_{n-1}}, W_{D_{n}}$ or $W_{B_{n}}$. The multiplication of the subalgebras $\mathbb{C}\left[\mathfrak{h}^{*}\right]$, $\mathbb{C}[\mathfrak{h}], \mathcal{C}_{\mathfrak{h}^{*}}, \mathcal{C}_{\mathfrak{h}}$, and $\mathbb{C} W$ induces a vector space isomorphism

$$
\mathbb{C}\left[\mathfrak{h}^{*}\right] \otimes \mathcal{C}_{\mathfrak{h}^{*}} \otimes \mathbb{C} W \otimes \mathbb{C}[\mathfrak{h}] \otimes \mathcal{C}_{\mathfrak{h}} \stackrel{\simeq}{\longrightarrow} \mathbb{H}_{W}^{c c}
$$

Equivalently, the elements $\left\{x^{\alpha} c^{\epsilon} w e^{\epsilon^{\prime}} y^{\gamma} \mid \alpha, \gamma \in \mathbb{Z}_{+}^{n}, \epsilon, \epsilon^{\prime} \in \mathbb{Z}_{2}^{n}, w \in W\right\}$ form a linear basis for $\mathbb{H}_{W}^{c c}$ (the PBW basis).

Proof. Recall that $W$ acts diagonally on $V=\mathfrak{h}^{*} \oplus \mathfrak{h}$. The strategy of proving the theorem follows the suggestion of [16] to modify [5, Proof of Theorem 1.3] as follows.

Clearly $K:=\mathcal{C}_{2 n} \rtimes \mathbb{C} W$ is a semisimple algebra. Observe that $E:=V \otimes_{\mathbb{C}} K$ is a natural $K$-bimodule (even though $V$ is not) with the right $K$-module structure on $E$ given by right multiplication and the left $K$-module structure on $E$ by letting

$$
\begin{aligned}
& w \cdot(v \otimes a)=v^{w} \otimes w a, \\
& c_{i} \cdot\left(x_{j} \otimes a\right)=(-1)^{\delta_{i j}} x_{j} \otimes\left(c_{i} a\right), \\
& c_{i} \cdot\left(y_{j} \otimes a\right)=y_{j} \otimes\left(c_{i} a\right), \\
& e_{i} \cdot\left(x_{j} \otimes a\right)=x_{j} \otimes\left(e_{i} a\right), \\
& e_{i} \cdot\left(y_{j} \otimes a\right)=(-1)^{\delta_{i j}} y_{j} \otimes\left(e_{i} a\right),
\end{aligned}
$$

where $v \in V, w \in W, a \in K$. 
The rest of the proof can proceed in the same way as in [5, Proof of Theorem 1.3], and it boils down to the verifications of the conjugation invariance (by $c_{i}, e_{i}$ and $W$ ) of the defining relations (3.2a)-(3.2b), (3.3a)-(3.3b), or (3.4a)-(3.4b) for type $A, D$ or $B$ respectively, and the verification of the Jacobi identities among the generators $x_{i}$ and $y_{i}$ for $1 \leq i \leq n$.

Such verifications are left to Lemmas 3.6, 3.7 and 3.8 below.

Remark 3.5. The algebra $\mathbb{H}_{W}^{c c}$ has two different triangular decompositions:

$$
\begin{aligned}
& \mathbb{H}_{W}^{c c} \cong \mathbb{C}\left[\mathfrak{h}^{*}\right] \otimes\left(\mathcal{C}_{2 n} \rtimes \mathbb{C} W\right) \otimes \mathbb{C}[\mathfrak{h}], \\
& \mathbb{H}_{W}^{c c} \cong\left(\mathbb{C}\left[\mathfrak{h}^{*}\right] \otimes \mathcal{C}_{\mathfrak{h}^{*}}\right) \otimes \mathbb{C} W \otimes\left(\mathcal{C}_{\mathfrak{h}} \otimes \mathbb{C}[\mathfrak{h}]\right) .
\end{aligned}
$$

The detailed proofs of Lemmas 3.6, 3.7 and 3.8 below (also compare [10]) are postponed to the Appendix.

Lemma 3.6. Let $W=W_{A_{n-1}}, W_{D_{n}}$ or $W_{B_{n}}$. Then the relations (3.2a) $-(3.2 \mathrm{~b})$, (3.3a)-(3.3b), or $(3.4 \mathrm{a})-(3.4 \mathrm{~b})$ are invariant under the conjugation by $c_{i}$ and $e_{i}$ respectively, $1 \leq i \leq n$.

Lemma 3.7. The relations (3.2a)-(3.2b), (3.3a)-(3.3b), or (3.4a)-(3.4b) are invariant under the conjugation by elements in $W_{A_{n-1}}, W_{D_{n}}$ or $W_{B_{n}}$ respectively.

Lemma 3.8. Let $W=W_{A_{n-1}}, W_{D_{n}}$ or $W_{B_{n}}$. Then the Jacobi identity holds for any triple among $x_{i}, y_{i}$ in $\mathbb{H}_{W}^{c c}$ for $1 \leq i \leq n$.

Remark 3.9. For $W=W_{A_{n-1}}, W_{D_{n}}$ or $W_{B_{n}}$, the algebra $\mathbb{H}_{W}^{c c}$ has a natural superalgebra structure by letting $x_{i}, y_{i}, s_{j}$ be even and $c_{k}, e_{k}$ be odd for all admissible $i, j, k$. Moreover, the map $\varpi: \mathbb{H}_{W}^{c c} \longrightarrow \mathbb{H}_{W}^{c c}$ which sends

$$
x_{i} \mapsto y_{i}, \quad y_{i} \mapsto-x_{i}, \quad c_{i} \mapsto e_{i}, \quad e_{i} \mapsto-c_{i}, \quad s_{j} \mapsto s_{j} \quad \forall i, j
$$

is an automorphism of $\mathbb{H}_{W}^{c c}$.

\subsection{The Dunkl representations}

Recall $K=\mathcal{C}_{2 n} \rtimes \mathbb{C} W$. Denote by $\mathfrak{H}_{y}$ the subalgebra of $\mathbb{H}_{W}^{c c}$ generated by $K$ and $y_{1}, \ldots, y_{n}$. A $K$-module $M$ can be extended to $\mathfrak{H}_{y}$-module by demanding the action of each $y_{i}$ to be trivial. We define

$$
M_{y}:=\operatorname{Ind}_{\mathfrak{H}_{y}}^{\mathbb{H}_{W}^{c c}} M
$$

Under the identification of vector spaces

$$
M_{y}=\mathbb{C}\left[x_{1}, \ldots, x_{n}\right] \otimes M,
$$

the action of $\mathbb{H}_{W}^{c c}$ on $M_{y}$ is transferred to $\mathbb{C}\left[x_{1}, \ldots, x_{n}\right] \otimes M$ as follows. $\mathrm{K}$ acts on $\mathbb{C}\left[x_{1}, \ldots, x_{n}\right] \otimes M$ by the following formulas:

$$
\begin{aligned}
& w \cdot\left(x_{j} \otimes m\right)=x_{j}^{w} \otimes w m, \\
& c_{i} \cdot\left(x_{j} \otimes m\right)=(-1)^{\delta_{i j}} x_{j} \otimes c_{i} m, \\
& e_{i} \cdot\left(x_{j} \otimes m\right)=x_{j} \otimes e_{i} m,
\end{aligned}
$$

where $c_{i}, e_{i} \in \mathcal{C}_{2 n}, w \in W$. Moreover, $x_{i}$ acts by left multiplication in the first tensor factor, and the action of $y_{i}$ will be given by the so-called Dunkl operators which we compute below (compare [3, 4]).

A simple choice for a $K$-module is $\mathcal{C}_{2 n}$, whose $K$-module structure is defined by letting $\mathcal{C}_{2 n}$ act by left multiplication and $W$ act diagonally. 


\subsubsection{The Dunkl Operators for type $A_{n-1}$ case}

We first prepare a few lemmas. It is understood in this paper that the ratios of two (possibly noncommutative) operators $g$ and $h$ always means that $\frac{h}{g}=\frac{1}{g} \cdot h$.

Lemma 3.10. Let $W=W_{A_{n-1}}$. Then the following holds in $\mathbb{H}_{W}^{c c}$ for $l \in \mathbb{Z}_{+}$and $i \neq j$ :

$$
\begin{aligned}
& {\left[y_{i}, x_{j}^{l}\right]=u\left(\frac{x_{j}^{l}-x_{i}^{l}}{x_{j}-x_{i}}+\frac{x_{j}^{l}-(-1)^{l} x_{i}^{l}}{x_{j}+x_{i}} c_{i} c_{j}\right)\left(1-e_{i} e_{j}\right) s_{i j},} \\
& {\left[y_{i}, x_{i}^{l}\right]=t c_{i} e_{i} \frac{x_{i}^{l}-\left(-x_{i}\right)^{l}}{2 x_{i}}-u \sum_{k \neq i}\left(\frac{x_{i}^{l}-x_{k}^{l}}{x_{i}-x_{k}}+\frac{x_{i}^{l}-\left(-x_{k}\right)^{l}}{x_{i}+x_{k}} c_{k} c_{i}\right)\left(1+e_{k} e_{i}\right) s_{k i} .}
\end{aligned}
$$

Proof. This lemma is a type $A$ counterpart of Lemma 3.13 for type $B$ below. A proof can be simply obtained by modifying the proof of Lemma 3.13 with the removal of those terms involving $\bar{s}_{i j}, \bar{s}_{k i}, \tau_{i}$ therein.

Lemma 3.11. Let $W=W_{A_{n-1}}$, and $f \in \mathbb{C}\left[x_{1}, \ldots, x_{n}\right]$. Then the following identity holds in $\mathbb{H}_{W}^{c c}$ :

$$
\left[y_{i}, f\right]=t c_{i} e_{i} \frac{f-f^{\tau_{i}}}{2 x_{i}}-u \sum_{k \neq i}\left(\frac{f-f^{s_{k i}}}{x_{i}-x_{k}}+\frac{f c_{k} c_{i}-c_{k} c_{i} f^{s_{k i}}}{x_{i}+x_{k}}\right)\left(1+e_{k} e_{i}\right) s_{k i} .
$$

Proof. It suffices to check the formula for every monomial $f$ of the form $x_{1}^{l_{1}} \cdots x_{n}^{l_{n}}$, which follows by Lemma 3.10 and an induction on $a$ based on the identity

$$
\left[y_{i}, x_{1}^{l_{1}} \cdots x_{a}^{l_{a}} x_{a+1}^{l_{a+1}}\right]=\left[y_{i}, x_{1}^{l_{1}} \cdots x_{a}^{l_{a}}\right] x_{a+1}^{l_{a+1}}+x_{1}^{l_{1}} \cdots x_{a}^{l_{a}}\left[y_{i}, x_{a+1}^{l_{a+1}}\right] .
$$

Now we are ready to compute the Dunkl operator for $y_{i}$.

Theorem 3.12. Let $W=W_{A_{n-1}}$ and $M$ be a $\left(\mathcal{C}_{2 n} \rtimes \mathbb{C} W\right)$-module. The action of $y_{i}$ on the module $\mathbb{C}\left[x_{1}, \ldots, x_{n}\right] \otimes M$ is realized as the following Dunkl operators: for any $f \in \mathbb{C}\left[x_{1}, \ldots, x_{n}\right]$ and $m \in M$, we have

$$
y_{i} \circ(f \otimes m)=t c_{i} e_{i} \frac{f-f^{\tau_{i}}}{2 x_{i}} \otimes m-u \sum_{k \neq i}\left(\frac{f-f^{s_{k i}}}{x_{i}-x_{k}}+\frac{f c_{k} c_{i}-c_{k} c_{i} f^{s_{k i}}}{x_{i}+x_{k}}\right) \otimes\left(1+e_{k} e_{i}\right) s_{k i} m .
$$

Proof. We calculate that

$$
y_{i} \circ(f \otimes m)=\left[y_{i}, f\right] \otimes m+f \otimes y_{i} m=\left[y_{i}, f\right] \otimes m .
$$

Now the result follows from Lemma 3.11 .

\subsubsection{The Dunkl Operators for type $B_{n}$ case}

The proofs of Lemmas 3.13 and 3.14 are postponed to the Appendix.

Lemma 3.13. Let $W=W_{B_{n}}$. Then the following holds in $\mathbb{H}_{W}^{c c}$ for $l \in \mathbb{Z}_{+}$and $i \neq j$ :

$$
\begin{aligned}
{\left[y_{i}, x_{j}^{l}\right]=} & u\left(\frac{x_{j}^{l}-x_{i}^{l}}{x_{j}-x_{i}}+\frac{x_{j}^{l}-(-1)^{l} x_{i}^{l}}{x_{j}+x_{i}} c_{i} c_{j}\right)\left(1-e_{i} e_{j}\right) s_{i j} \\
& -u\left(\frac{x_{j}^{l}-\left(-x_{i}\right)^{l}}{x_{j}+x_{i}}-\frac{x_{j}^{l}-x_{i}^{l}}{x_{j}-x_{i}} c_{i} c_{j}\right)\left(1+e_{i} e_{j}\right) \bar{s}_{i j},
\end{aligned}
$$




$$
\begin{aligned}
{\left[y_{i}, x_{i}^{l}\right]=} & t c_{i} e_{i} \frac{x_{i}^{l}-\left(-x_{i}\right)^{l}}{2 x_{i}}-u \sum_{k \neq i}\left(\frac{x_{i}^{l}-x_{k}^{l}}{x_{i}-x_{k}}+\frac{x_{i}^{l}-\left(-x_{k}\right)^{l}}{x_{i}+x_{k}} c_{k} c_{i}\right)\left(1+e_{k} e_{i}\right) s_{k i} \\
& -u \sum_{k \neq i}\left(\frac{x_{i}^{l}-\left(-x_{k}\right)^{l}}{x_{i}+x_{k}}-\frac{x_{i}^{l}-x_{k}^{l}}{x_{i}-x_{k}} c_{k} c_{i}\right)\left(1-e_{k} e_{i}\right) \bar{s}_{k i}-v \frac{x_{i}^{l}-\left(-x_{i}\right)^{l}}{2 x_{i}} \tau_{i} .
\end{aligned}
$$

Lemma 3.14. Let $W=W_{B_{n}}$, and $f \in \mathbb{C}\left[x_{1}, \ldots, x_{n}\right]$. Then the following holds in $\mathbb{H}_{W}^{c c}$ :

$$
\begin{aligned}
{\left[y_{i}, f\right]=} & t c_{i} e_{i} \frac{f-f^{\tau_{i}}}{2 x_{i}}-u \sum_{k \neq i}\left(\frac{f-f^{s_{k i}}}{x_{i}-x_{k}}+\frac{f-f^{\bar{s}_{k i}}}{x_{i}+x_{k}} c_{k} c_{i}\right)\left(1+e_{k} e_{i}\right) s_{k i} \\
& -v \frac{f-f^{\tau_{i}}}{2 x_{i}} \tau_{i}-u \sum_{k \neq i}\left(\frac{f-f^{\bar{s}_{k i}}}{x_{i}+x_{k}}-\frac{f-f^{s_{k i}}}{x_{i}-x_{k}} c_{k} c_{i}\right)\left(1-e_{k} e_{i}\right) \bar{s}_{k i} .
\end{aligned}
$$

Now we are ready to compute the Dunkl operator for $y_{i}$.

Theorem 3.15. Let $W=W_{B_{n}}$ and $M$ be $a\left(\mathcal{C}_{2 n} \rtimes \mathbb{C} W\right)$-module. The action of $y_{i}$ on the module $\mathbb{C}\left[x_{1}, \ldots, x_{n}\right] \otimes M$ is realized as the following Dunkl operators: for any $f \in \mathbb{C}\left[x_{1}, \ldots, x_{n}\right]$ and $m \in M$, we have

$$
\begin{aligned}
y_{i} \circ(f \otimes m)= & t c_{i} e_{i} \frac{f-f^{\tau_{i}}}{2 x_{i}} \otimes m-u \sum_{k \neq i}\left(\frac{f-f^{s_{k i}}}{x_{i}-x_{k}}+\frac{f-f^{\bar{s}_{k i}}}{x_{i}+x_{k}} c_{k} c_{i}\right) \otimes\left(1+e_{k} e_{i}\right) s_{k i} m \\
& -u \sum_{k \neq i}\left(\frac{f-f^{\bar{s}_{k i}}}{x_{i}+x_{k}}-\frac{f-f^{s_{k i}}}{x_{i}-x_{k}} c_{k} c_{i}\right) \otimes\left(1-e_{k} e_{i}\right) \bar{s}_{k i} m-v \frac{f-f^{\tau_{i}}}{2 x_{i}} \otimes \tau_{i} m .
\end{aligned}
$$

Proof. We observe that

$$
y_{i} \circ(f \otimes m)=\left[y_{i}, f\right] \otimes m+f \otimes y_{i} m=\left[y_{i}, f\right] \otimes m .
$$

Now the result follows from Lemma 3.14.

\subsubsection{The Dunkl Operators for type $D_{n}$ case}

Due to the similarity of the bracket relations [-, - ] in $D_{n}$ and $B_{n}$ cases (e.g. compare (3.3b) with (3.4b)), the formula below for type $D_{n}$ is obtained from its type $B_{n}$ counterpart in the previous subsection by dropping the terms involving the parameter $v$.

Theorem 3.16. Let $W=W_{D_{n}}$, and let $M$ be a $\left(\mathcal{C}_{2 n} \rtimes \mathbb{C} W\right)$-module. The action of $y_{i}$ on $\mathbb{C}\left[x_{1}, \ldots, x_{n}\right] \otimes M$ is realized as the following Dunkl operators: for any $f \in \mathbb{C}\left[x_{1}, \ldots, x_{n}\right]$ and $m \in M$, we have

$$
\begin{aligned}
y_{i} \circ(f \otimes m)= & t c_{i} e_{i} \frac{f-f^{\tau_{i}}}{2 x_{i}} \otimes m-u \sum_{k \neq i}\left(\frac{f-f^{s_{k i}}}{x_{i}-x_{k}}+\frac{f-f^{\bar{s}_{k i}}}{x_{i}+x_{k}} c_{k} c_{i}\right) \otimes\left(1+e_{k} e_{i}\right) s_{k i} m \\
& -u \sum_{k \neq i}\left(\frac{f-f^{\bar{s}_{k i}}}{x_{i}+x_{k}}-\frac{f-f^{s_{k i}}}{x_{i}-x_{k}} c_{k} c_{i}\right) \otimes\left(1-e_{k} e_{i}\right) \bar{s}_{k i} m .
\end{aligned}
$$

\subsection{The even center for $\mathbb{H}_{W}^{c c}$}

Recall that the even center $\mathcal{Z}(A)$ of a superalgebra $A$ consists of the even central elements of $A$. It turns out the algebra $\mathbb{H}_{W}^{c c}$ has a large center. 
Proposition 3.17. Let $W$ be either $W_{A_{n-1}}, W_{D_{n}}$ or $W_{B_{n}}$. The even center $\mathcal{Z}\left(\mathbb{H}_{W}^{c c}\right)$ contains $\mathbb{C}\left[x_{1}^{2}, \ldots, x_{n}^{2}\right]^{W}$ and $\mathbb{C}\left[y_{1}^{2}, \ldots, y_{n}^{2}\right]^{W}$ as subalgebras. In particular, $\mathbb{H}_{W}^{c c}$ is module-finite over its even center.

Proof. Let $f \in \mathbb{C}\left[x_{1}^{2}, \ldots, x_{n}^{2}\right]^{W}$. Then $f-f^{\tau_{i}}=0$ for each $i$. Moreover, by the definition of $\mathbb{H}_{W}^{c c}$, $f$ commutes with $\mathcal{C}_{2 n}, W$, and $x_{i}$ for all $1 \leq i \leq n$. Since $f=f^{w}$ for all $w \in W$, it follows from Lemmas 3.11 and 3.14 that $\left[y_{i}, f\right]=0$ for each $i$. Hence $f$ commutes with $\mathbb{C}\left[y_{1}, \ldots, y_{n}\right]$. Therefore $f$ is in the even center $\mathcal{Z}\left(\mathbb{H}_{W}^{c c}\right)$. It follows from the automorphism $\varpi$ of $\mathbb{H}_{W}^{c c}$ defined in Remark 3.9 that $\mathbb{C}\left[y_{1}^{2}, \ldots, y_{n}^{2}\right]^{W}$ must also be in the even center $\mathcal{Z}\left(\mathbb{H}_{W}^{c c}\right)$.

\section{The spin double affine Hecke--Clifford algebras}

Recall that $W$ is one of the classical Weyl groups of type $A_{n-1}, B_{n}$, or $D_{n}$. The goal of this section is to introduce and study the spin double affine Hecke-Clifford algebra (sDaHCa) $\mathbb{H}_{W}^{-c}$, which is, roughly speaking, obtained by decoupling the Clifford algebra $\mathcal{C}_{\mathfrak{h}}$ from the DaHa $\mathbb{H}_{W}^{c c}$ in Section 3. The spin Weyl group algebra $\mathbb{C} W^{-}$appears naturally in the process. We remark that the algebra $\mathbb{H}_{W}^{-c}$ is different from either the spin double affine Hecke algebra or the double affine Hecke-Clifford algebra introduced in [16, 10].

\subsection{The definition of sDaHCa $\mathbb{H}_{W}^{-c}$}

Following [10], we introduce the notation

$$
t_{i \uparrow j}=\left\{\begin{array}{ll}
t_{i} t_{i+1} \cdots t_{j}, & \text { if } i \leq j, \\
1, & \text { otherwise }
\end{array} \quad t_{i \downarrow j}= \begin{cases}t_{i} t_{i-1} \cdots t_{j}, & \text { if } i \geq j \\
1, & \text { otherwise }\end{cases}\right.
$$

Define the following odd elements in $\mathbb{C} W^{-}$of order 2 , which are an analogue of reflections in $W$, for $1 \leq i<j \leq n$ :

$$
\begin{aligned}
& t_{i j} \equiv[i, j]=(-1)^{j-i-1} t_{j-1} \cdots t_{i+1} t_{i} t_{i+1} \cdots t_{j-1}, \\
& t_{j i} \equiv[j, i]=-[i, j], \\
& \bar{t}_{i j} \equiv \overline{[i, j]}= \begin{cases}(-1)^{j-i-1} t_{j \uparrow n-1} t_{i \uparrow n-2} t_{n} t_{n-2 \downarrow i} t_{n-1 \downarrow j}, & \text { for type } D_{n}, \\
(-1)^{j-i} t_{j \uparrow n-1} t_{i \uparrow n-2} t_{n} t_{n-1} t_{n} t_{n-2 \downarrow i} t_{n-1 \downarrow j}, & \text { for type } B_{n},\end{cases} \\
& \bar{t}_{j i} \equiv \overline{[j, i]}=\overline{[i, j]}, \\
& \bar{t}_{i} \equiv \overline{[i]}=(-1)^{n-i} t_{i} \cdots t_{n-1} t_{n} t_{n-1} \cdots t_{i} \quad(1 \leq i \leq n) .
\end{aligned}
$$

The notations $[i, j], \overline{[i, j]}$ here are consistent with the inclusions of algebras $\mathbb{C} W_{A_{n-1}}^{-} \leq \mathbb{C} W_{D_{n}}^{-} \leq$ $\mathbb{C} W_{B_{n}}^{-}$.

As in [16] (also cf. $[9,10]$ ), a skew-polynomial algebra is the $\mathbb{C}$-algebra generated by $b_{1}, \ldots, b_{n}$ subject to the relations $b_{i} b_{j}+b_{j} b_{i}=0, \quad(i \neq j)$. This algebra, denoted by $\mathcal{C}\left[b_{1}, \ldots, b_{n}\right]$, is naturally a superalgebra by letting each $b_{i}$ be odd, and it has a linear basis given by $b^{\alpha}:=$ $b_{1}^{k_{1}} \cdots b_{n}^{k_{n}}$ for $\alpha=\left(k_{1}, \ldots, k_{n}\right) \in \mathbb{Z}_{+}^{n}$.

Consider the group homomorphism $\rho: W_{B_{n}} \rightarrow S_{n}$ defined by $\rho\left(s_{i}\right)=s_{i}$ and $\rho\left(s_{n}\right)=1$ for $1 \leq i \leq n-1$. By restriction if needed, we have a group homomorphism

$$
\rho: W \longrightarrow S_{n}, \quad \sigma \mapsto \rho(\sigma)=\sigma^{*}
$$

for $W=W_{A_{n-1}}, W_{B_{n}}$ or $W_{D_{n}}$. Observe that $\tau_{i}^{*}=1$ and $\bar{s}_{i j}^{*}=s_{i j}$ for all $1 \leq i \neq j \leq n$. 
Definition 4.1. Let $t, u, v \in \mathbb{C}$, and $W=W_{A_{n-1}}, W_{D_{n}}$, or $W_{B_{n}}$. The sDaHCa $\mathbb{H}_{W}^{-c}$ is the algebra generated by $x_{i}, \eta_{i}(1 \leq i \leq n)$ and $\mathcal{C}_{\mathfrak{h}^{*}} \rtimes \mathbb{C} W^{-}$, subject to the relations

$$
\begin{aligned}
& \eta_{i} \eta_{j}=-\eta_{j} \eta_{i}, \quad x_{i} x_{j}=x_{j} x_{i} \quad(i \neq j), \\
& c_{i} \eta_{j}=-\eta_{j} c_{i}, \quad c_{i} x_{j}=(-1)^{\delta_{i j}} x_{j} c_{i} \quad(\forall i, j), \\
& t_{i} x_{j}=x_{j}^{s_{i}} t_{i}, \quad t_{i} \eta_{j}=-\eta_{j}^{s_{i}^{*}} t_{i} \quad\left(t_{i} \in \mathbb{C} W^{-}\right)
\end{aligned}
$$

and the following additional relations:

$$
\begin{array}{ll}
\text { Type A: }\left\{\begin{array}{l}
{\left[\eta_{i}, x_{j}\right]=u\left(1+c_{i} c_{j}\right)[i, j] \quad(i \neq j),} \\
{\left[\eta_{i}, x_{i}\right]=t c_{i}+u \sum_{k \neq i}\left(1+c_{k} c_{i}\right)[k, i],}
\end{array}\right. \\
\text { Type D: } \begin{cases}{\left[\eta_{i}, x_{j}\right]=u\left(\left(1+c_{i} c_{j}\right)[i, j]-\left(1-c_{i} c_{j}\right) \overline{[i, j]}\right) \quad(i \neq j),} \\
{\left[\eta_{i}, x_{i}\right]=t c_{i}+u \sum_{k \neq i}\left(\left(1+c_{k} c_{i}\right)[k, i]-\left(1-c_{k} c_{i}\right)[\overline{[k, i]}),\right.}\end{cases} \\
\text { Type B: } \begin{cases}{\left[\eta_{i}, x_{j}\right]=u\left(\left(1+c_{i} c_{j}\right)[i, j]-\left(1-c_{i} c_{j}\right) \overline{[i, j]}\right)} & (i \neq j), \\
{\left[\eta_{i}, x_{i}\right]=t c_{i}+u \sum_{k \neq i}\left(\left(1+c_{k} c_{i}\right)[k, i]-\left(1-c_{k} c_{i}\right) \overline{[k, i]}\right)+v \overline{[i]} .}\end{cases}
\end{array}
$$

\subsection{Isomorphism of superalgebras}

For $W=W_{A_{n-1}}, W_{B_{n}}$, or $W_{D_{n}}$, we recall an algebra isomorphism (see [10, Lemma 5.4])

$\Phi: \mathcal{C}_{\mathfrak{h}} \rtimes \mathbb{C} W \rightarrow \mathcal{C}_{\mathfrak{h}} \otimes \mathbb{C} W^{-}$

which sends

$$
\begin{aligned}
& \left(e_{k}-e_{i}\right) s_{i k} \longmapsto-\sqrt{-2}[k, i], \\
& \left(e_{k}+e_{i}\right) \bar{s}_{i k} \longmapsto-\sqrt{-2}[k, i], \\
& e_{i} \tau_{i} \longmapsto-\sqrt{-1} \overline{[i]}
\end{aligned}
$$

for $i \neq k$, whenever it is applicable. The inverse of $\Phi$ is denoted by $\Psi$.

Note that the algebra $\mathbb{H}_{W}^{-c}$ has a natural superalgebra structure by letting each $\eta_{i}, c_{i}, t_{j}$ be odd and $x_{i}$ be even for all admissible $i, j$.

Theorem 4.2. Let $W$ be $W_{A_{n-1}}, W_{D_{n}}$ or $W_{B_{n}}$. Then,

1) there exists an isomorphism of superalgebras

$$
\Phi: \mathbb{H}_{W}^{c c}(t, u, v) \longrightarrow \mathcal{C}_{\mathfrak{h}} \otimes \mathbb{H}_{W}^{-c}(-t,-\sqrt{-2} u, \sqrt{-1} v)
$$

which extends $\Phi: \mathcal{C}_{\mathfrak{h}} \rtimes \mathbb{C} W \rightarrow \mathcal{C}_{\mathfrak{h}} \otimes \mathbb{C} W^{-}$and sends

$$
y_{i} \mapsto e_{i} \eta_{i}, \quad x_{i} \mapsto x_{i}, \quad c_{i} \mapsto c_{i}, \quad \forall i ;
$$

2) the inverse

$$
\Psi: \mathcal{C}_{\mathfrak{h}} \otimes \mathbb{H}_{W}^{-c}(-t,-\sqrt{-2} u, \sqrt{-1} v) \longrightarrow \mathbb{H}_{W}^{c c}(t, u, v)
$$

extends $\Psi: \mathcal{C}_{\mathfrak{h}} \otimes \mathbb{C} W^{-} \rightarrow \mathcal{C}_{\mathfrak{h}} \rtimes \mathbb{C} W$ and sends

$$
\eta_{i} \mapsto e_{i} y_{i}, \quad x_{i} \mapsto x_{i}, \quad c_{i} \mapsto c_{i}, \quad \forall i
$$


Proof. We need to check that $\Phi$ preserves the relations (3.1), (3.2a)-(3.2b), (3.3a)-(3.3b), and (3.4a)-(3.4b) for $W=W_{A_{n-1}}, W_{D_{n}}$, and $W_{B_{n}}$ respectively.

First, we shall verify that $\Phi$ preserves (3.4a)-(3.4b) with $W=W_{B_{n}}$. Indeed, by (4.1) or [10, Lemma 5.4], we have

$$
\begin{aligned}
& \Phi(\text { l.h.s. of }(3.4 \mathrm{a}))=e_{i}\left[\eta_{i}, x_{j}\right]=-\sqrt{-2} u e_{i}\left(\left(1-c_{j} c_{i}\right)[i, j]-\left(1+c_{j} c_{i}\right) \overline{[i, j]}\right) \\
& \quad=\Phi\left(u\left(\left(1+c_{i} c_{j}\right)\left(1+e_{j} e_{i}\right) s_{j i}-\left(1-c_{i} c_{j}\right)\left(1-e_{j} e_{i}\right) \bar{s}_{i j}\right)\right) \\
& =\Phi \text { (r.h.s. of }(3.4 \mathrm{a})) .
\end{aligned}
$$

Also, we have

$$
\begin{aligned}
& \Phi(\text { l.h.s. of }(3.4 \mathrm{~b}))=e_{i}\left[\eta_{i}, x_{i}\right] \\
& =-t \cdot e_{i} c_{i}-\sqrt{-2} u e_{i} \sum_{k \neq i}\left(\left(1+c_{k} c_{i}\right)[k, i]-\left(1-c_{k} c_{i}\right) \overline{[k, i]}\right)+\sqrt{-1} v e_{i} \overline{[i]} \\
& =\Phi\left(t c_{i} e_{i}-u \sum_{k \neq i}\left(\left(1+c_{k} c_{i}\right)\left(1+e_{k} e_{i}\right) s_{k i}+\left(1-c_{k} c_{i}\right)\left(1-e_{k} e_{i}\right) \bar{s}_{k i}\right)-v \tau_{i}\right) \\
& =\Phi \text { (r.h.s. of }(3.4 \mathrm{~b})) .
\end{aligned}
$$

It is easy to check that $\Phi$ preserves (3.1), and we will restrict ourselves to verify just a few relations among (3.1). For $j \neq i, i+1$, we have

$$
\Phi\left(s_{i} y_{j}\right)=-\sqrt{-1} \nu_{i} t_{i} e_{j} \eta_{j}=-\sqrt{-1} e_{j} \eta_{j} \nu_{i} t_{i}=\Phi\left(y_{j} s_{i}\right) .
$$

Moreover,

$$
\Phi\left(s_{n} y_{n}\right)=-\sqrt{-1} \nu_{n} t_{n} e_{n} \eta_{n}=\sqrt{-1} t_{n} \eta_{n}=-\sqrt{-1} \eta_{n} t_{n}=\Phi\left(-y_{n} s_{n}\right) .
$$

This proves that $\Phi$ is an algebra homomorphism for type $B_{n}$.

By dropping the terms involving $v$ in the above equations, we verify that the relations (3.3a)(3.3b) with $W=W_{D_{n}}$ are preserved by $\Phi$. By further dropping the terms involving $\overline{[i j]}$, $\bar{s}_{i j}$ etc., we also verify (3.2a)-(3.2b) with $W=W_{A_{n-1}}$. So, the homomorphism $\Phi$ is well defined in all cases.

Similarly, one shows that $\Psi$ is a well-defined algebra homomorphism. Since $\Phi$ and $\Psi$ are inverses on generators, they are (inverse) algebra isomorphisms.

The isomorphism in Theorem 4.2 exactly means that the superalgebras $\mathbb{H}_{W}^{c c}$ and $\mathbb{H}_{W}^{-c}$ are Morita super-equivalent in the sense of [17].

Corollary 4.3. Let $W$ be one of the Weyl groups $W_{A_{n-1}}, W_{D_{n}}$ or $W_{B_{n}}$. The even center $\mathcal{Z}\left(\mathbb{H}_{W}^{-c}\right)$ of $\mathbb{H}_{W}^{-c}$ contains $\mathbb{C}\left[\eta_{1}^{2}, \ldots, \eta_{n}^{2}\right]^{W}$ and $\mathbb{C}\left[x_{1}^{2}, \ldots, x_{n}^{2}\right]^{W}$. In particular, $\mathbb{H}_{W}^{-c}$ is module-finite over its even center.

Proof. By the isomorphism $\Phi$ in Theorem 4.2 and the Proposition 3.17, we have that $\mathcal{Z}\left(\mathcal{C}_{\mathfrak{h}} \otimes\right.$ $\left.\mathbb{H}_{W}^{-c}\right)$ contains the subalgebras $\mathbb{C}\left[\eta_{1}^{2}, \ldots, \eta_{n}^{2}\right]^{W}$ and $\mathbb{C}\left[x_{1}^{2}, \ldots, x_{n}^{2}\right]^{W}$, and so does $\mathcal{Z}\left(\mathbb{H}_{W}^{-c}\right)$.

\subsection{The PBW property for $\mathbb{H}_{W}^{-c}$}

We have the following PBW type property for the algebra $\mathbb{H}_{W}^{-c}$.

Theorem 4.4. Let $W$ be one of the Weyl groups $W_{A_{n-1}}, W_{D_{n}}$ or $W_{B_{n}}$. The multiplication of the subalgebras induces an isomorphism of vector spaces

$$
\mathcal{C}\left[\eta_{1}, \ldots, \eta_{n}\right] \otimes \mathcal{C}_{\mathfrak{h}^{*}} \otimes \mathbb{C} W^{-} \otimes \mathbb{C}\left[\mathfrak{h}^{*}\right] \longrightarrow \mathbb{H}_{W}^{-c}
$$

Equivalently, the set $\left\{\eta^{\alpha} c^{\epsilon} \sigma x^{\gamma}\right\}$ forms a basis for $\mathbb{H}_{W}^{-c}$, where $\sigma$ runs over a basis for $\mathbb{C W}^{-}$, $\epsilon \in \mathbb{Z}_{2}^{n}$, and $\alpha, \gamma \in \mathbb{Z}_{+}^{n}$. 
Proof. It follows from the defining relations that $\mathbb{H}_{W}^{-c}$ is spanned by the elements $\eta^{\alpha} c^{\epsilon} \sigma x^{\gamma}$ where $\sigma$ runs over a basis for $\mathbb{C} W^{-}, \alpha, \gamma \in \mathbb{Z}_{+}^{n}$, and $\epsilon \in \mathbb{Z}_{2}^{n}$. By the isomorphism $\Psi: \mathcal{C}_{\mathfrak{h}} \otimes \mathbb{H}_{W}^{-c} \longrightarrow \mathbb{H}_{W}^{c c}$ in Theorem 4.2, we see that the image $\Psi\left(\eta^{\alpha} c^{\epsilon} \sigma x^{\gamma}\right)$ are linearly independent in $\mathbb{H}_{W}^{c c}$ by the PBW property for $\mathbb{H}_{W}^{c c}$ (see Theorem 3.4). So the elements $\eta^{\alpha} c^{\epsilon} \sigma x^{\gamma}$ are linearly independent in $\mathbb{H}_{W}^{-c}$. Therefore, the set $\left\{\eta^{\alpha} c^{\epsilon} \sigma x^{\gamma}\right\}$ forms a basis for $\mathbb{H}_{W}^{-c}$.

\subsection{The Dunkl operators for $\mathbb{H}_{W}^{-c}$}

Denote by $\mathfrak{h}_{\eta}$ the subalgebra of $\mathbb{H}_{W}^{-c}$ generated by $\eta_{i}(1 \leq i \leq n)$ and $\mathcal{C}_{\mathfrak{h}^{*}} \rtimes_{-} \mathbb{C} W^{-}$. A $\left(\mathcal{C}_{\mathfrak{h}^{*}} \rtimes_{-}\right.$ $\mathbb{C} W^{-}$)-module $V$ can be extended to a $\mathfrak{h}_{\eta}$-modules by letting the actions of $\eta_{i}$ on $V$ to be trivial for each $i$. We define

$$
V_{\eta}:=\operatorname{Ind}_{\mathfrak{h}_{\eta}}^{\mathbb{H}_{W}^{-c}} V \cong \mathbb{C}\left[x_{1}, \ldots, x_{n}\right] \otimes V .
$$

On $\mathbb{C}\left[x_{1}, \ldots, x_{n}\right] \otimes V$, the element $t_{i} \in \mathbb{C} W^{-}$acts as $s_{i} \otimes t_{i}, c_{i} \in \mathcal{C}_{\mathfrak{h}^{*}}$ acts by $c_{i} \cdot\left(x_{j} \otimes v\right)=$ $(-1)^{\delta_{i j}} x_{j} \otimes c_{i} v$, and $x_{i}$ acts by left multiplication, and $\eta_{i}$ acts as anti-commuting Dunkl operators, which we will describe in this section.

Under the superalgebra isomorphism $\Phi: \mathbb{H}_{W}^{c c} \rightarrow \mathcal{C}_{n} \otimes \mathbb{H}_{W}^{-c}$ in Theorem 4.2, we obtain anticommuting Dunkl operators $\eta_{i}$ by fairly straightforward computation. They are counterparts of those in Section 3, and we omit the proofs.

\subsubsection{Dunkl operator for type $A_{n-1}$}

The following is a counterpart of Theorem 3.12.

Proposition 4.5. Let $W=W_{A_{n-1}}$ and $V$ be a $\mathcal{C}_{n} \rtimes \mathbb{C} W^{-}$-module. The action of $\eta_{i}$ on the $\mathbb{H}_{W}^{-c}$-module $\mathbb{C}\left[x_{1}, \ldots, x_{n}\right] \otimes V$ is realized as a Dunkl operator as follows. For any polynomial $f \in \mathbb{C}\left[x_{1}, \ldots, x_{n}\right]$ and $m \in V$, we have

$$
\eta_{i} \circ(f \otimes m)=t c_{i} \frac{f-f^{\tau_{i}}}{2 x_{i}} \otimes m+u \sum_{k \neq i}\left(\frac{f-f^{s_{k i}}}{x_{i}-x_{k}}+\frac{f c_{k} c_{i}-c_{k} c_{k} f^{s_{k i}}}{x_{i}+x_{k}}\right) \otimes[k, i] m .
$$

\subsubsection{Dunkl operator for type $B_{n}$}

The following is a counterpart of Theorem 3.15

Proposition 4.6. Let $W=W_{B_{n}}$ and $V$ be a $\left(\mathcal{C}_{n} \rtimes \mathbb{C} W^{-}\right)$-module. The action of $\eta_{i}$ on the $\mathbb{H}_{W}^{-c}$-module $\mathbb{C}\left[x_{1}, \ldots, x_{n}\right] \otimes V$ is realized as a Dunkl operator as follows. For any polynomial $f \in \mathbb{C}\left[x_{1}, \ldots, x_{n}\right]$ and $m \in V$, we have

$$
\begin{aligned}
\eta_{i} \circ(f \otimes m)= & t c_{i} \frac{f-f^{\tau_{i}}}{2 x_{i}} \otimes m+u \sum_{k \neq i}\left(\frac{f-f^{s_{k i}}}{x_{i}-x_{k}}+\frac{f-f^{\bar{s}_{k i}}}{x_{i}+x_{k}} c_{k} c_{i}\right) \otimes[k, i] m \\
& -u \sum_{k \neq i}\left(\frac{f-f^{\bar{s}_{k i}}}{x_{i}+x_{k}}-\frac{f-f^{s_{k i}}}{x_{i}-x_{k}} c_{k} c_{i}\right) \otimes \overline{[k, i]} m+v \frac{f-f^{\tau_{i}}}{2 x_{i}} \otimes \overline{[i]} m .
\end{aligned}
$$

\subsubsection{Dunkl operator for type $D_{n}$}

Proposition 4.7. Let $W=W_{D_{n}}$ and $V$ be a $\mathcal{C}_{n} \rtimes \mathbb{C} W^{-}$-module. The action of $\eta_{i}$ on the $\mathbb{H}_{W}^{-c}$-module $\mathbb{C}\left[x_{1}, \ldots, x_{n}\right] \otimes V$ is realized as a Dunkl operator as follows. For any polynomial $f \in \mathbb{C}\left[x_{1}, \ldots, x_{n}\right]$ and $m \in V$, we have

$$
\eta_{i} \circ(f \otimes m)=t c_{i} \frac{f-f^{\tau_{i}}}{2 x_{i}} \otimes m+u \sum_{k \neq i}\left(\frac{f-f^{s_{k i}}}{x_{i}-x_{k}}+\frac{f-f^{\bar{s}_{k i}}}{x_{i}+x_{k}} c_{k} c_{i}\right) \otimes[k, i] m
$$




$$
-u \sum_{k \neq i}\left(\frac{f-f^{\bar{s}_{k i}}}{x_{i}+x_{k}}-\frac{f-f^{s_{k i}}}{x_{i}-x_{k}} c_{k} c_{i}\right) \otimes \overline{[k, i]} m .
$$

Remark 4.8. The general formula for the Dunkl operators $\eta_{i}$ for $\mathbb{H}_{W}^{-c}$ resembles the Dunkl operator $y_{i}$ for $\ddot{\mathfrak{H}}_{W}^{\mathfrak{c}}$ which appeared in [10, Theorems 4.4, 4.10, 4.14]. However, $\eta_{i} \eta_{j}=-\eta_{j} \eta_{i}$, while $y_{i} y_{j}=y_{j} y_{i}$ for $i \neq j$.

\section{The odd double affine Hecke algebras}

In this section, we shall introduce an odd double affine Hecke algebra $\mathbb{H}_{W}$ which is generated by $\mathbb{C} W$ and two isomorphic skew-polynomial subalgebras. Recall that $W$ is assumed to be one of the classical Weyl groups of type $A_{n-1}, B_{n}$, or $D_{n}$.

Recall also the group homomorphism $\rho: W \longrightarrow S_{n}$ defined in Section 4 which sends $\sigma \mapsto \sigma^{*}$ for all $\sigma \in W$. We shall need two (isomorphic) skew-polynomial algebras $\mathcal{C}\left\{\mathfrak{h}^{*}\right\}=\mathcal{C}\left[\xi_{1}, \ldots, \xi_{n}\right]$ and $\mathcal{C}\{\mathfrak{h}\}=\mathcal{C}\left[\eta_{1}, \ldots, \eta_{n}\right]$, which are naturally acted upon by the symmetric group $S_{n}$ or the group $W_{B_{n}}$ by permuting the indices possibly coupled with sign changes. We shall denote the action of $\sigma \in W_{B_{n}}$ by $f \mapsto f^{\sigma}$.

\subsection{The definition of $\mathbb{H}_{W}$}

As usual we denote $[\xi, \eta]_{+}=\xi \eta+\eta \xi$.

Definition 5.1. Let $t, u, v \in \mathbb{C}$ and $W$ be $W_{A_{n-1}}, W_{D_{n}}$, or $W_{B_{n}}$. The odd DaHa $\mathbb{H}_{W}$ is the algebra generated by $\xi_{i}, \eta_{i}(1 \leq i \leq n)$ and $\mathbb{C} W$, subject to the relations

$$
\begin{aligned}
& \eta_{i} \eta_{j}=-\eta_{j} \eta_{i}, \quad \xi_{i} \xi_{j}=-\xi_{j} \xi_{i} \quad(i \neq j), \\
& \sigma \xi_{j}=\xi_{j}^{\sigma^{*}} \sigma, \quad \sigma \eta_{j}=\eta_{j}^{\sigma^{*}} \sigma \quad(\sigma \in W)
\end{aligned}
$$

and the following additional relations:

$$
\begin{array}{ll}
\text { Type A: } & \left\{\begin{array}{l}
{\left[\eta_{i}, \xi_{j}\right]_{+}=u s_{i j} \quad(i \neq j),} \\
{\left[\eta_{i}, \xi_{i}\right]_{+}=t \cdot 1+u \sum_{k \neq i} s_{k i},}
\end{array}\right. \\
\text { Type D: } \begin{cases}{\left[\eta_{i}, \xi_{j}\right]_{+}=u\left(s_{i j}+\bar{s}_{i j}\right)} & (i \neq j), \\
{\left[\eta_{i}, \xi_{i}\right]_{+}=t \cdot 1+u \sum_{k \neq i}\left(s_{k i}+\bar{s}_{i j}\right),}\end{cases} \\
\text { Type B: } \begin{cases}{\left[\eta_{i}, \xi_{j}\right]_{+}=u\left(s_{i j}+\bar{s}_{i j}\right)} & (i \neq j), \\
{\left[\eta_{i}, \xi_{i}\right]_{+}=t \cdot 1+u \sum_{k \neq i}\left(s_{k i}+\bar{s}_{i j}\right)+v \tau_{i} .}\end{cases}
\end{array}
$$

The algebra $\mathbb{H}_{W}$ has a natural superalgebra structure by letting $s_{j}$ be even and $\eta_{i}, \xi_{i}$ be odd for all $i, j$.

Remark 5.2. The defining relations for the algebra $\mathbb{H}_{W}$ differ from those for the usual rational DaHa (also known as rational Cherednik algebra) $\ddot{\mathfrak{H}}_{W}[5]$ by signs. One can introduce a so-called "covering algebra" $\widetilde{\mathbb{H}}$ (as done in $[17,10]$ in similar setups) which contains a central element $z$ of order 2 , so that the algebras $\ddot{\mathfrak{H}}_{W}$ and $\mathbb{H}_{W}$ are simply the quotients of $\widetilde{\mathbb{H}}$ by the ideal generated by $z-1$ and $z+1$ respectively.

The definition of $\mathbb{H}_{W}$ is motivated by the Morita (super)equivalence with $\mathbb{H}_{W}^{c c}$ and $\mathbb{H}_{W}^{-c}$. The defining relations above suggest a further extension of odd DaHa associated to the infinite series complex reflection groups. 


\subsection{Isomorphism of superalgebras}

Lemma 5.3. Let $W$ be one of the Weyl groups $W_{A_{n-1}}, W_{D_{n}}$ or $W_{B_{n}}$. The isomorphism $\dot{\Phi}$ : $\mathcal{C}_{n} \rtimes_{-} \mathbb{C} W^{-} \rightarrow \mathcal{C}_{n} \otimes \mathbb{C} W$ (see Theorem 2.3) sends

$$
\left(c_{k}-c_{i}\right)[k, i] \longmapsto \sqrt{2} s_{k i}, \quad\left(c_{k}+c_{i}\right) \overline{[k, i]} \longmapsto \sqrt{2} \bar{s}_{i k}, \quad c_{i} \overline{[i]} \longmapsto \tau_{i} .
$$

Proof. The lemma can be proved by induction very similar to [10, Lemma 5.4], and we skip the detail.

Theorem 5.4. Let $W$ be one of the Weyl groups $W_{A_{n-1}}, W_{D_{n}}$ or $W_{B_{n}}$. Then,

1) there exists an isomorphism of superalgebras

$$
\dot{\Phi}: \mathbb{H}_{W}^{-c}(t, u, v) \longrightarrow \mathcal{C}_{n} \otimes \mathbb{H}_{W}(-t, \sqrt{2} u,-v)
$$

which extends $\dot{\Phi}: \mathcal{C}_{n} \rtimes_{-} \mathbb{C} W^{-} \rightarrow \mathcal{C}_{n} \otimes \mathbb{C} W$ and sends

$$
\eta_{i} \mapsto \eta_{i}, \quad x_{i} \mapsto c_{i} \xi_{i}, \quad \forall i ;
$$

2) the inverse

$$
\dot{\Psi}: \mathcal{C}_{n} \otimes \mathbb{H}_{W}(-t, \sqrt{2} u,-v) \longrightarrow \mathbb{H}_{W}^{-c}(t, u, v)
$$

extends $\dot{\Psi}: \mathcal{C}_{n} \otimes \mathbb{C} W \rightarrow \mathcal{C}_{n} \rtimes_{-} \mathbb{C} W^{-}$and sends

$$
\eta_{i} \mapsto \eta_{i}, \quad \xi_{i} \mapsto c_{i} x_{i}, \quad \forall i
$$

Proof. We first need to check that $\dot{\Phi}$ preserves the defining relations of $\mathbb{H}_{W}^{-c}(t, u, v)$ and so $\Phi$ is a well-defined homomorphism. Using Lemma 5.3, we shall check a few cases in type $B_{n}$ case, and leave the rest for the reader to verify. For $i \neq j$, we have

$$
\begin{aligned}
\dot{\Phi}\left(\left[\eta_{i}, x_{j}\right]\right) & =-c_{j}\left[\eta_{i}, \xi_{j}\right]_{+}=-\sqrt{2} u c_{j}\left(s_{i j}+\bar{s}_{i j}\right) \\
& =\frac{u}{\sqrt{2}}\left(\left(1+c_{i} c_{j}\right)\left(c_{i}-c_{j}\right) s_{i j}-\left(1-c_{i} c_{j}\right)\left(c_{i}+c_{j}\right) \bar{s}_{i j}\right) \\
& =\dot{\Phi}\left(u\left(\left(1+c_{i} c_{j}\right)[i, j]-\left(1-c_{i} c_{j}\right) \overline{[i, j]}\right)\right), \\
\dot{\Phi}\left(\left[\eta_{i}, x_{i}\right]\right) & =-c_{i}\left[\eta_{i}, \xi_{i}\right]_{+}=-c_{i}\left(-t+\sqrt{2} u \sum_{k \neq i}\left(s_{i k}+\bar{s}_{i k}\right)-v \tau_{i}\right) \\
& =t c_{i}-\sqrt{2} u c_{i} \sum_{k \neq i}\left(s_{i k}+\bar{s}_{i k}\right)+v c_{i} \tau_{i} \\
& =\dot{\Phi}\left(t c_{i}+u \sum_{k \neq i}\left(\left(1+c_{k} c_{i}\right)[k, i]+\left(1-c_{k} c_{i}\right) \overline{[k, i]}\right)+v \tau_{i}\right) .
\end{aligned}
$$

Also, if $j \neq n$, we have

$$
\begin{aligned}
& \dot{\Phi}\left(t_{n} x_{j}\right)=c_{n} s_{n} c_{j} \xi_{j}=c_{j} \xi_{j} c_{n} s_{n}=\dot{\Phi}\left(x_{j} t_{n}\right), \\
& \dot{\Phi}\left(t_{n} x_{n}\right)=c_{n} s_{n} c_{n} \xi_{n}=s_{n} \xi_{n}=\dot{\Phi}\left(-x_{n} t_{n}\right), \\
& \dot{\Phi}\left(t_{n} \eta_{j}\right)=c_{n} s_{n} \eta_{j}=-\eta_{j} c_{n} s_{n}=\dot{\Phi}\left(-\eta_{j} t_{n}\right), \\
& \dot{\Phi}\left(t_{n} \eta_{n}\right)=c_{n} s_{n} \eta_{n}=-\eta_{n} c_{n} s_{n}=\dot{\Phi}\left(-\eta_{n} t_{n}\right) .
\end{aligned}
$$

Similarly, one shows that $\dot{\Psi}$ is a well-defined algebra homomorphism. Since $\dot{\Phi}$ and $\dot{\Psi}$ are inverses on generators, they are (inverse) algebra isomorphisms. 
The next corollary can be proved similarly to Corollary 4.3.

Corollary 5.5. Let $W$ be one of the Weyl groups $W_{A_{n-1}}, W_{D_{n}}$ or $W_{B_{n}}$. The even center for $\mathbb{H}_{W}$ contains $\mathbb{C}\left[\eta_{1}^{2}, \ldots, \eta_{n}^{2}\right]^{W}$ and $\mathbb{C}\left[\xi_{1}^{2}, \ldots, \xi_{n}^{2}\right]^{W}$. In particular, $\mathbb{H}_{W}$ is module-finite over its even center.

Example 5.6. Usually there are other central elements beyond those given in the above corollary. For example, $\xi_{1}^{2} \eta_{2}^{2}+\xi_{2}^{2} \eta_{1}^{2}-u s_{1}\left(\xi_{1}-\xi_{2}\right)\left(\eta_{1}-\eta_{2}\right)$ lies in $\mathcal{Z}\left(\mathbb{H}_{W_{A_{1}}}\right)$.

\subsection{The PBW property for $\mathbb{H}_{W}$}

We have the following PBW type property for the algebra $\mathbb{H}_{W}$ which can be proved similarly to Theorem 4.4, using now the isomorphism $\dot{\Phi}$.

Theorem 5.7. Let $W$ be one of the Weyl groups $W_{A_{n-1}}, W_{D_{n}}$ or $W_{B_{n}}$. The multiplication of the subalgebras induces an isomorphism of vector spaces

$$
\mathcal{C}\left[\xi_{1}, \ldots, \xi_{n}\right] \otimes \mathbb{C} W \otimes \mathcal{C}\left[\eta_{1}, \ldots, \eta_{n}\right] \longrightarrow \mathbb{H}_{W} .
$$

Equivalently, the set $\left\{\xi^{\alpha} \sigma \eta^{\gamma}\right\}$ forms a basis for $\mathbb{H}_{W}$, where $\sigma \in W$, and $\alpha, \gamma \in \mathbb{Z}_{+}^{n}$.

\subsection{The Dunkl operators for $\mathbb{H}_{W}$}

Denote by $\mathfrak{h}_{\eta}$ the subalgebra of $\mathbb{H}_{W}$ generated by $\eta_{i}(1 \leq i \leq n)$ and $\mathbb{C} W$. Let $V$ be the trivial $\mathbb{C} W$-module, and extend $V$ a $\mathfrak{h}_{\eta}$-module by letting the actions of each $\eta_{i}$ on $\mathrm{V}$ be trivial. Define

$$
V_{\eta}:=\operatorname{Ind}_{\mathfrak{h}_{\eta}}^{\mathbb{H}_{W}} V \cong \mathcal{C}\left[\xi_{1}, \ldots, \xi_{n}\right] .
$$

On $\mathcal{C}\left[\xi_{1}, \ldots, \xi_{n}\right], \sigma \in W$ acts as $\rho(\sigma)=\sigma^{*}, \xi_{i}$ acts by left multiplication, and $\eta_{i}$ acts as anticommuting Dunkl operators which we establish below. (It is easy to replace the trivial module above by any $\mathbb{C} W$-module.)

\subsubsection{Dunkl operator for type $A$ case}

For each $i$, we introduce a super derivation $\partial_{\xi_{i}}$ on $\mathcal{C}\left[\xi_{1}, \ldots, \xi_{n}\right]$ defined inductively by $\partial_{\xi_{i}}\left(\xi_{j}\right)=\delta_{i j}$ and

$$
\partial_{\xi_{i}}\left(\xi_{a_{1}} \cdots \xi_{a_{l}}\right)=\sum_{k}(-1)^{k-1} \xi_{a_{1}} \cdots \xi_{a_{k-1}} \partial_{\xi_{i}}\left(\xi_{a_{k}}\right) \xi_{a_{k+1}} \cdots \xi_{a_{l}} .
$$

The formulas below for type $A_{n-1}$ case can be obtained from Lemmas 5.11, 5.12, and Theorem 5.13 with the removal of those terms involving $\bar{s}_{i j}, \bar{s}_{k i}$, and the parameter $v$ therein.

Lemma 5.8. Let $W=W_{A_{n-1}}$. Then the following holds in $\mathbb{H}_{W}$ for $l \in \mathbb{Z}_{+}$and $i \neq j$ :

$$
\begin{aligned}
& {\left[\eta_{i}, \xi_{j}^{l}\right]_{+}=\frac{u}{\xi_{i}^{2}-\xi_{j}^{2}}\left(\xi_{i}^{l+1}-\xi_{j} \xi_{i}^{l}-\xi_{i} \xi_{j}^{l}+(-1)^{l} \xi_{j}^{l+1}\right) s_{i j},} \\
& {\left[\eta_{i}, \xi_{i}^{l}\right]_{+}=t \frac{\xi_{i}^{l}-\left(-\xi_{i}\right)^{l}}{2 \xi_{i}}+u \sum_{k \neq i} \frac{1}{\xi_{i}^{2}-\xi_{k}^{2}}\left(\xi_{i} \xi_{k}^{l}-\xi_{k}^{l+1}-(-1)^{l} \xi_{i}^{l+1}+\xi_{k} \xi_{i}^{l}\right) s_{i k} .}
\end{aligned}
$$

Lemma 5.9. Let $W=W_{A_{n-1}}$, and $f \in \mathcal{C}\left[\xi_{1}, \ldots, \xi_{n}\right]$. Then the following identity holds in $\mathbb{H}_{W}$ :

$$
\left[\eta_{i}, f\right]_{+}=t \frac{f-f^{\tau_{i}}}{2 \xi_{i}}+u \sum_{k \neq i} \frac{1}{\xi_{i}^{2}-\xi_{k}^{2}}\left(\left(\xi_{i}-\xi_{k}\right) f^{s_{i k}}-\left(\xi_{i} f^{\tau_{i}}-\xi_{k} f^{\tau_{k}}\right)\right) s_{k i} .
$$

Theorem 5.10. Let $W=W_{A_{n-1}}$. The action of $\eta_{i}$ on $\mathcal{C}\left[\xi_{1}, \ldots, \xi_{n}\right]$ is realized as Dunkl operators as follows:

$$
\eta_{i}=t \partial_{\xi_{i}}+u \sum_{k \neq i} \frac{1}{\xi_{i}^{2}-\xi_{k}^{2}}\left(\left(\xi_{i}-\xi_{k}\right) s_{i k}-\left(\xi_{i} \tau_{i}-\xi_{k} \tau_{k}\right)\right) .
$$




\subsubsection{Dunkl operator for type $B_{n}$ case}

The proofs of Lemma 5.11 and 5.12 are given in the Appendix.

Lemma 5.11. Let $W=W_{B_{n}}$. Then the following holds in $\mathbb{H}_{W}$ for $l \in \mathbb{Z}_{+}$and $i \neq j$ :

$$
\begin{aligned}
{\left[\eta_{i}, \xi_{j}^{l}\right]_{+}=} & u\left(\xi_{i}^{l-1}-\xi_{j} \xi_{i}^{l-2}+\cdots+(-1)^{l-1} \xi_{j}^{l-1}\right)\left(s_{i j}+\bar{s}_{i j}\right) \\
= & u\left(\frac{1}{\xi_{i}^{2}-\xi_{j}^{2}}\left(\xi_{i}^{l+1}-\xi_{j} \xi_{i}^{l}-\xi_{i} \xi_{j}^{l}+(-1)^{l} \xi_{j}^{l+1}\right)\right)\left(s_{i j}+\bar{s}_{i j}\right), \\
{\left[\eta_{i}, \xi_{i}^{l}\right]_{+}=} & t \frac{\xi_{i}^{l}-\left(-\xi_{i}\right)^{l}}{2 \xi_{i}}+v \frac{\xi_{i}^{l}-\left(-\xi_{i}\right)^{l}}{2 \xi_{i}} \tau_{i} \\
& +u \sum_{k \neq i}\left(\xi_{k}^{l-1}-\xi_{i} \xi_{k}^{l-2}+\cdots+(-1)^{l-1} \xi_{i}^{l-1}\right)\left(s_{i k}+\bar{s}_{i k}\right) \\
= & t \frac{\left.\xi_{i}^{l}-\left(-\xi_{i}\right)^{l}\right)}{2 \xi_{i}}+v \frac{\xi_{i}^{l}-\left(-\xi_{i}\right)^{l}}{2 \xi_{i}} \tau_{i} \\
& +u \sum_{k \neq i} \frac{1}{\xi_{i}^{2}-\xi_{k}^{2}}\left(\xi_{i} \xi_{k}^{l}-\xi_{k}^{l+1}-(-1)^{l} \xi_{i}^{l+1}+\xi_{k} \xi_{i}^{l}\right)\left(s_{i k}+\bar{s}_{i k}\right) .
\end{aligned}
$$

Lemma 5.12. Let $W=W_{B_{n}}$, and $f \in \mathcal{C}\left[\xi_{1}, \ldots, \xi_{n}\right]$. Then the following identity holds in $\mathbb{H}_{W}$ :

$$
\begin{aligned}
{\left[\eta_{i}, f\right]_{+}=} & t \frac{f-f^{\tau_{i}}}{2 \xi_{i}}+v \frac{f-f^{\tau_{i}}}{2 \xi_{i}} \tau_{i} \\
& +u \sum_{k \neq i} \frac{1}{\xi_{i}^{2}-\xi_{k}^{2}}\left(\left(\xi_{i}-\xi_{k}\right) f^{s_{i k}}-\left(\xi_{i} f^{\tau_{i}}-\xi_{k} f^{\tau_{k}}\right)\right)\left(s_{i k}+\bar{s}_{i k}\right) .
\end{aligned}
$$

Theorem 5.13. Let $W=W_{B_{n}}$. The action of $\eta_{i}$ on $\mathcal{C}\left[\xi_{1}, \ldots, \xi_{n}\right]$ is realized as operators as follows:

$$
\eta_{i}=t \partial_{\xi_{i}}+v \frac{1-\tau_{i}}{2 \xi_{i}}+u \sum_{k \neq i} \frac{2}{\xi_{i}^{2}-\xi_{k}^{2}}\left(\left(\xi_{i}-\xi_{k}\right) s_{i k}-\left(\xi_{i} \tau_{i}-\xi_{k} \tau_{k}\right)\right)
$$

Proof. It suffices to check the formula for every monomial $f$. Consider $f=\xi_{1}^{a_{1}} \cdots \xi_{n}^{a_{n}}$ where $a_{i} \in \mathbb{Z}_{+}$, and observe that

$$
\partial_{\xi_{i}}(f)=\frac{f-f^{\tau_{i}}}{2 \xi_{i}}, \quad \eta_{i} \cdot f=\left[\eta_{i}, f\right]_{+}+(-1)^{a_{1}+\cdots+a_{n}} f \cdot \eta_{i}=\left[\eta_{i}, f\right]_{+} .
$$

The theorem now follows by Lemma 5.12.

\subsubsection{Dunkl operator for type $D_{n}$ case}

The formula below for the Dunkl operator type $D_{n}$ case is obtained from their type $B_{n}$ counterparts (see Theorem 5.13) by dropping the terms involving the parameter $v$.

Theorem 5.14. Let $W=W_{D_{n}}$. The action of $\eta_{i}$ on $\mathcal{C}\left[\xi_{1}, \ldots, \xi_{n}\right]$ is realized as Dunkl operators as follows:

$$
\eta_{i}=t \partial_{\xi_{i}}+u \sum_{k \neq i} \frac{2}{\xi_{i}^{2}-\xi_{k}^{2}}\left(\left(\xi_{i}-\xi_{k}\right) s_{i k}-\left(\xi_{i} \tau_{i}-\xi_{k} \tau_{k}\right)\right) .
$$

Remark 5.15. Let $W=W_{A_{n-1}}, W_{B_{n}}$, or $W_{D_{n}}$. The Dunkl operators $\eta_{i}$ anti-commute, i.e. $\eta_{i} \eta_{j}=-\eta_{j} \eta_{i}(i \neq j)$. It is not easy to check this directly. 


\subsection{An affine Hecke subalgebra}

In this subsection, we will show that the odd DaHa of type $A$ contains as a subalgebra the degenerate affine Hecke algebra of type $A$ introduced by Drinfeld and Lusztig $[2,11]$. Let

$$
\mathfrak{z}_{i}=-\xi_{i} \eta_{i}+u \sum_{k<i} s_{k i}
$$

Lemma 5.16. We have $\left[\mathfrak{z}_{i}, \mathfrak{z}_{j}\right]=0, \forall i, j$.

Proof. Let us assume $i<j$. Then,

$$
\begin{aligned}
{\left[\hat{\mathfrak{z}}_{i}, \mathfrak{z}_{j}\right] } & =\left[-\xi_{i} \eta_{i},-\xi_{j} \eta_{j}+u \sum_{k<j} s_{k j}\right]=\left(\xi_{i}\left[\eta_{i}, \xi_{j}\right]_{+} \eta_{j}-\xi_{j}\left[\eta_{j}, \xi_{i}\right]_{+} \eta_{i}\right)-u\left[\xi_{i} \eta_{i}, s_{i j}\right] \\
& =u\left(\xi_{i} s_{i j} \eta_{j}-\xi_{j} s_{i j} \eta_{i}\right)-u\left(\xi_{i} \eta_{i}-\xi_{j} \eta_{j}\right) s_{i j}=0 .
\end{aligned}
$$

Lemma 5.17. The following identities hold:

$$
s_{i} \mathfrak{z}_{i}=\mathfrak{z}_{i+1} s_{i}-u, \quad s_{i} \mathfrak{z}_{j}=\mathfrak{z}_{j} s_{i} \quad(j \neq i, i+1) .
$$

Proof. Recall that $L_{i}:=\sum_{k<i} s_{k i}$ is the Jucys-Murphy element, and it is known that $s_{i} L_{i}=$ $L_{i+1} s_{i}-1$ and $s_{i} L_{j}=L_{j} s_{i}$ for $j \neq i, i+1$. The lemma follows from these relations.

Proposition 5.18. The $\mathfrak{z}_{i}(1 \leq i \leq n)$ and $S_{n}$ generate the degenerate affine Hecke algebra.

Proof. The proposition follows from Theorem 5.7 and Lemma 5.17.

\section{A Appendix: proofs of several lemmas}

\section{A.1 Proofs of Lemmas in Section 3}

\section{A.1.1 Proof of Lemma 3.6}

We will show that the relations (3.4a) and (3.4b) are invariant under the conjugation by elements $c_{l}$ and $e_{l}, 1 \leq l \leq n$. We will only verify for the $c_{l}$ and leave the similar verification for the $e_{l}$ to the reader. Also, the verifications for the invariants in type $A$ and $D$ under the conjugation by $c_{l}$ and $e_{l}$ are similar and will be omitted.

Consider the relation (3.4a) first. Clearly, (3.4a) is invariant under the conjugation by $c_{l}$, and $e_{l}$ if $l \neq i, j$. Moreover, we calculate that

$$
\begin{aligned}
c_{i}(\text { r.h.s. of }(3.4 \mathrm{a})) c_{i} & =u\left(\left(1+c_{i} c_{j}\right)\left(1+e_{j} e_{i}\right) s_{i j}-\left(1-c_{i} c_{j}\right)\left(1-e_{j} e_{i}\right) \bar{s}_{i j}\right) \\
& =\left[y_{i}, x_{j}\right]=c_{i}(\text { l.h.s. of }(3.4 \mathrm{a})) c_{i}, \\
c_{j}(\text { r.h.s. of }(3.4 \mathrm{a})) c_{j} & =u\left(\left(c_{j} c_{i}-1\right)\left(1+e_{j} e_{i}\right) s_{j i}-\left(-c_{j} c_{i}-1\right)\left(1-e_{j} e_{i}\right) \bar{s}_{i j}\right) \\
& \left.=-\left[y_{i}, x_{j}\right]=c_{j} \text { (l.h.s. of }(3.4 \mathrm{a})\right) c_{j} .
\end{aligned}
$$

Thus, (3.4a) is conjugation-invariant by all $c_{l}$.

Next, we will show that the relation $(3.4 \mathrm{~b})$ is invariant under the conjugation by each $c_{l}$. Indeed, we have

$$
\begin{aligned}
& c_{i}(\text { r.h.s. of }(3.4 \mathrm{~b})) c_{i} \\
& \quad=t e_{i} c_{i}-v c_{i} \tau_{i} c_{i}-u \sum_{k \neq i} c_{i}\left(\left(1+c_{k} c_{i}\right)\left(1+e_{k} e_{i}\right) s_{k i}+\left(1-c_{k} c_{i}\right)\left(1-e_{k} e_{i}\right) \bar{s}_{k i}\right) c_{i}
\end{aligned}
$$




$$
\begin{aligned}
& =-t c_{i} e_{i}+v \tau_{i}-u \sum_{k \neq i}\left(\left(c_{i} c_{k}-1\right)\left(1+e_{k} e_{i}\right) s_{k i}+\left(-c_{i} c_{k}-1\right)\left(1-e_{k} e_{i}\right) \bar{s}_{k i}\right) \\
& =-t c_{i} e_{i}+v \tau_{i}+u \sum_{k \neq i}\left(\left(1+c_{k} c_{i}\right)\left(1+e_{k} e_{i}\right) s_{k i}+\left(1-c_{k} c_{i}\right)\left(1-e_{k} e_{i}\right) \bar{s}_{k i}\right) \\
& =-\left[y_{i}, x_{i}\right]=c_{i}(\text { l.h.s. of }(3.4 \mathrm{~b})) c_{i} .
\end{aligned}
$$

For $j \neq i$, we have

$$
\begin{aligned}
& c_{j}(\text { r.h.s. of }(3.4 \mathrm{~b})) c_{j} \\
& =t c_{i} e_{i}-v \tau_{i}-u c_{j}\left(\left(1+c_{j} c_{i}\right)\left(1+e_{j} e_{i}\right) s_{j i}+\left(1-c_{j} c_{i}\right)\left(1-e_{j} e_{i}\right) \bar{s}_{j i}\right) c_{j} \\
& \quad-u \sum_{k \neq i, j} c_{j}\left(\left(1+c_{k} c_{i}\right)\left(1+e_{k} e_{i}\right) s_{k i}+\left(1-c_{k} c_{i}\right)\left(1-e_{k} e_{i}\right) \bar{s}_{k i}\right) c_{j} \\
& =t c_{i} e_{i}-v \tau_{i}-u\left(\left(c_{j} c_{i}+1\right)\left(1+e_{j} e_{i}\right) s_{j i}+\left(-c_{j} c_{i}+1\right)\left(1-e_{j} e_{i}\right) \bar{s}_{j i}\right) c_{j} \\
& \quad-u \sum_{k \neq i, j}\left(\left(1+c_{k} c_{i}\right)\left(1+e_{k} e_{i}\right) s_{k i}+\left(1-c_{k} c_{i}\right)\left(1-e_{k} e_{i}\right) \bar{s}_{k i}\right) \\
& \left.=c_{j} \text { (l.h.s. of }(3.4 \mathrm{~b})\right) c_{j} .
\end{aligned}
$$

Therefore, the lemma is proved.

\section{A.1.2 Proof of Lemma 3.7}

We will show below that the relations $(3.4 \mathrm{a})-(3.4 \mathrm{~b})$ are invariant under the conjugation by elements in $W_{B_{n}}$. The proof can be readily modified to yield the Weyl group invariance of the relations (3.2a)-(3.2b) and (3.3a)-(3.3b) in type $A$ and $D$ cases respectively, and we leave the details to the reader.

(i) We check the invariance of (3.4a) under $W_{B_{n}}$.

Consider first the conjugation invariance by the transposition $s_{l k}$. If $\{l, k\} \cap\{i, j\}=\varnothing$, then we have

$$
\begin{aligned}
s_{l k}(\text { r.h.s. of }(3.4 \mathrm{a})) s_{l k} & =u\left(\left(1+c_{i} c_{j}\right)\left(1+e_{j} e_{i}\right) s_{i j}-\left(1-c_{i} c_{j}\right)\left(1-e_{j} e_{i}\right) \bar{s}_{i j}\right) \\
& \left.=\left[y_{i}, x_{j}\right]=s_{l k} \text { (1.h.s. of }(3.4 \mathrm{a})\right) s_{l k} .
\end{aligned}
$$

If $\{l, k\} \cap\{i, j\}=\{j\}$, then we may assume $l=j$ and we have

$$
\begin{aligned}
\left.s_{j k} \text { (r.h.s. of }(3.4 \mathrm{a})\right) s_{j k} & =u\left(\left(1+c_{i} c_{k}\right)\left(1+e_{k} e_{i}\right) s_{i k}-\left(1-c_{i} c_{k}\right)\left(1-e_{k} e_{i}\right) \bar{s}_{i k}\right) \\
& =\left[y_{i}, x_{k}\right]=s_{j k}(\text { l.h.s. of }(3.4 \mathrm{a})) s_{j k} .
\end{aligned}
$$

We leave an entirely analogous computation when $\{l, k\} \cap\{i, j\}=\{i\}$ to the reader.

Now, if $\{l, k\}=\{i, j\}$, then

$$
\begin{aligned}
s_{i j}(\text { r.h.s. of }(3.4 \mathrm{a})) s_{i j} & =u\left(\left(1+c_{j} c_{i}\right)\left(1+e_{i} e_{j}\right) s_{i j}-\left(1-c_{j} c_{i}\right)\left(1-e_{i} e_{j}\right) \bar{s}_{i j}\right) \\
& =\left[y_{j}, x_{i}\right]=s_{i j}(\text { l.h.s. of }(3.4 \mathrm{a})) s_{i j} .
\end{aligned}
$$

So (3.4a) is invariant under the conjugation by each transposition $s_{l k}$.

It remains to show that $(3.4 \mathrm{a})$ is invariant under the conjugation by the simple reflection $s_{n}=\tau_{n}$. Observe that (3.4a) is clearly invariant under the conjugation by $s_{n}$ for $n \neq i, j$. Moreover, if $j=n$ then we have

$$
\begin{aligned}
s_{n}(\text { r.h.s. of }(3.4 \mathrm{a})) s_{n} & =u\left(\left(1-c_{i} c_{j}\right)\left(1-e_{j} e_{i}\right) \bar{s}_{i j}-\left(1+c_{i} c_{j}\right)\left(1+e_{j} e_{i}\right) s_{i j}\right) \\
& =-\left[y_{i}, x_{j}\right]=s_{n}(\text { l.h.s. of }(3.4 \mathrm{a})) s_{n} .
\end{aligned}
$$


If $i=n$, then we have

$$
\begin{aligned}
s_{n}(\text { r.h.s. of }(3.4 \mathrm{a})) s_{n} & =u\left(\left(1-c_{i} c_{j}\right)\left(1-e_{j} e_{i}\right) \bar{s}_{j i}-\left(1+c_{i} c_{j}\right)\left(1+e_{j} e_{i}\right) s_{i j}\right) \\
& =-\left[y_{i}, x_{j}\right]=s_{n}(\text { l.h.s. of }(3.4 \mathrm{a})) s_{n} .
\end{aligned}
$$

This completes (i).

(ii) We check the invariance of (3.4b) under $W_{B_{n}}$.

Consider first the conjugation invariance by $s_{j l}$. If $\{j, l\} \cap\{i\}=\varnothing$, then we have

$$
\begin{aligned}
& s_{j l}(\text { r.h.s. of }(3.4 b)) s_{j l} \\
& =t c_{i} e_{i}-v \tau_{i}-u \sum_{k \neq i, j, l} s_{j l}\left(\left(1+c_{k} c_{i}\right)\left(1+e_{k} e_{i}\right) s_{k i}+\left(1-c_{k} c_{i}\right)\left(1-e_{k} e_{i}\right) \bar{s}_{k i}\right) s_{j l} \\
& -u s_{j l}\left(\left(1+c_{j} c_{i}\right)\left(1+e_{j} e_{i}\right) s_{j i}+\left(1-c_{j} c_{i}\right)\left(1-e_{j} e_{i}\right) \bar{s}_{j i}\right) s_{j l} \\
& -u s_{j l}\left(\left(1+c_{l} c_{i}\right)\left(1+e_{l} e_{i}\right) s_{l i}+\left(1-c_{l} c_{i}\right)\left(1-e_{l} e_{i}\right) \bar{s}_{l i}\right) s_{j l} \\
& \left.=\left[y_{i}, x_{i}\right]=s_{j l} \text { (1.h.s. of }(3.4 \mathrm{~b})\right) s_{j l} \text {. }
\end{aligned}
$$

If $\{j, l\} \cap\{i\}=\{i\}$, we may assume that $j=i$, and then we have

$$
\begin{aligned}
& s_{i l}(\text { r.h.s. of }(3.4 b)) s_{i l} \\
& =t c_{l} e_{l}-u s_{i l}\left(\left(1+c_{l} c_{i}\right)\left(1+e_{l} e_{i}\right) s_{j i}+\left(1-c_{l} c_{i}\right)\left(1-e_{l} e_{i}\right) \bar{s}_{l i}\right) s_{i l} \\
& \quad-u \sum_{k \neq i, l} s_{i l}\left(\left(1+c_{k} c_{l}\right)\left(1+e_{k} e_{i}\right) s_{k l}+\left(1-c_{k} c_{l}\right)\left(1-e_{k} e_{i}\right) \bar{s}_{k l}\right) s_{i l}-v \tau_{l} \\
& \left.=\left[y_{l}, x_{l}\right]=s_{i l} \text { (1.h.s. of }(3.4 \mathrm{~b})\right) s_{i l} .
\end{aligned}
$$

It remains to show that $(3.4 \mathrm{~b})$ is invariant under the conjugation by the simple reflection $s_{n} \equiv \tau_{n} \in W_{B_{n}}$. If $i \neq n$, we have

$$
\begin{aligned}
& \left.s_{n} \text { (r.h.s. of }(3.4 b)\right) s_{n} \\
& \left.=t c_{i} e_{i}-v \tau_{i}-u s_{n}\left(\left(1+c_{n} c_{i}\right)\left(1+e_{n} e_{i}\right) s_{n i}+\left(1-c_{n} c_{i}\right)\left(1-e_{n} e_{i}\right) \bar{s}_{n i}\right)\right) s_{n} \\
& \quad-u \sum_{k \neq i, n} s_{n}\left(\left(1+c_{k} c_{i}\right)\left(1+e_{k} e_{i}\right) s_{k i}+\left(1-c_{k} c_{i}\right)\left(1-e_{k} e_{i}\right) \bar{s}_{k i}\right) s_{n} \\
& \left.=-v \tau_{i}-u\left(\left(1-c_{n} c_{i}\right) \bar{s}_{n i}+\left(1+c_{n} c_{i}\right) s_{n i}\right)\right)-u \sum_{k \neq i, n}\left(\left(1+c_{k} c_{i}\right) s_{k i}+\left(1-c_{k} c_{i}\right) \bar{s}_{k i}\right) \\
& =\left[y_{i}, x_{i}\right]=s_{n}(\text { l.h.s. of }(3.4 \mathrm{~b})) s_{n} .
\end{aligned}
$$

If $i=n$, then

$$
\begin{aligned}
& \left.s_{n} \text { (r.h.s. of }(3.4 b)\right) s_{n} \\
& \quad=t c_{n} e_{n}-v \tau_{n}-u \sum_{k \neq n}\left(\left(1-c_{k} c_{n}\right)\left(1-e_{k} e_{n}\right) \bar{s}_{k n}+\left(1+c_{k} c_{n}\right)\left(1+e_{k} e_{n}\right) s_{k n}\right) \\
& \quad=\left[y_{n}, x_{n}\right]=s_{n}(\text { l.h.s. of }(3.4 \mathrm{~b})) s_{n} .
\end{aligned}
$$

This completes the proof of (ii). Hence the lemma is proved.

\section{A.1.3 Proof of Lemma 3.8}

We will establish the Jacobi identity for $W=W_{B_{n}}$. The proof can be easily modified for the cases of type $A$ and $D$, and we leave the details to the reader. 
The Jacobi identity trivially holds among triple $x_{i}$ 's or triple $y_{i}$ 's.

Now, we consider the triple with two $y$ 's and one $x$. The case with two identical $y_{i}$ is trivial. So we first consider $x_{i}, y_{j}$, and $y_{l}$ where $i, j, l$ are all distinct. The Jacobi identity holds in this case since

$$
\begin{aligned}
{\left[x_{i},\left[y_{j}, y_{l}\right]\right]+\left[y_{l},\left[x_{i}, y_{j}\right]\right]+\left[y_{j},\left[y_{l}, x_{i}\right]\right] } \\
=0+\left[y_{l},-u\left(\left(1+c_{j} c_{i}\right)\left(1+e_{i} e_{j}\right) s_{j i}-\left(1-c_{j} c_{i}\right)\left(1-e_{i} e_{j}\right) \bar{s}_{i j}\right)\right] \\
\quad+\left[y_{j}, u\left(\left(1+c_{l} c_{i}\right)\left(1-e_{i} e_{l}\right) s_{l i}-\left(1-c_{l} c_{i}\right)\left(1-e_{i} e_{l}\right) \bar{s}_{i l}\right)\right]=0 .
\end{aligned}
$$

Now for $i \neq j$, we have

$$
\begin{aligned}
& {\left[x_{i},\left[y_{i}, y_{j}\right]\right]+\left[y_{j},\left[x_{i}, y_{i}\right]\right]+\left[y_{i},\left[y_{j}, x_{i}\right]\right] } \\
&=\left[y_{j},-t c_{i} e_{i}+u \sum_{k \neq i}\left(\left(1+c_{k} c_{i}\right)\left(1+e_{k} e_{i}\right) s_{k i}+\left(1-c_{k} c_{i}\right)\left(1-e_{k} e_{i}\right) \bar{s}_{k i}\right)+v \tau_{i}\right] \\
& \quad+\left[y_{i}, u\left(\left(1+c_{j} c_{i}\right)\left(1+e_{i} e_{j}\right) s_{i j}-\left(1-c_{j} c_{i}\right)\left(1-e_{i} e_{j}\right) \bar{s}_{i j}\right)\right] \\
&=\left[y_{j}, u \sum_{k \neq i, j}\left(\left(1+c_{k} c_{i}\right)\left(1+e_{k} e_{i}\right) s_{k i}+\left(1-c_{k} c_{i}\right)\left(1-e_{k} e_{i}\right) \bar{s}_{k i}\right)\right] \\
& \quad+\left[y_{j}, u\left(\left(1+c_{j} c_{i}\right)\left(1+e_{j} e_{i}\right) s_{j i}+\left(1-c_{j} c_{i}\right)\left(1-e_{j} e_{i}\right) \bar{s}_{j i}\right)\right] \\
& \quad+\left[y_{i}, u\left(\left(1+c_{j} c_{i}\right)\left(1+e_{i} e_{j}\right) s_{i j}-\left(1-c_{j} c_{i}\right)\left(1-e_{i} e_{j}\right) \bar{s}_{i j}\right)\right] \\
&=0+u\left(y_{j}\left(1+c_{j} c_{i}\right)\left(1+e_{j} e_{i}\right) s_{j i}+y_{j}\left(1-c_{j} c_{i}\right)\left(1-e_{j} e_{i}\right) \bar{s}_{j i}\right) \\
&-u\left(\left(1+c_{j} c_{i}\right)\left(1+e_{j} e_{i}\right) s_{j i} y_{j}+\left(1-c_{j} c_{i}\right)\left(1-e_{j} e_{i}\right) \bar{s}_{j i} y_{j}\right) \\
&+u\left(y_{i}\left(1+c_{j} c_{i}\right)\left(1+e_{i} e_{j}\right) s_{j i}-y_{i}\left(1-c_{j} c_{i}\right)\left(1-e_{i} e_{j}\right) \bar{s}_{i j}\right) \\
&-u\left(\left(1+c_{j} c_{i}\right)\left(1+e_{i} e_{j}\right) s_{j i} y_{i}-\left(1-c_{j} c_{i}\right)\left(1-e_{i} e_{j}\right) \bar{s}_{i j} y_{i}\right)=0 .
\end{aligned}
$$

Thanks to the automorphism $\varpi$ of $\mathbb{H}_{W}^{c c}$ which switches $x_{i}$ and $y_{i}$, we obtain the Jacobi identity with one $y$ and two $x$ 's from the above calculation. This completes the proof of Lemma 3.8.

\section{A.1.4 Proof of Lemma 3.13}

We will proceed by induction on $l$. For $l=1$, then the equations hold by (3.4a) and (3.4b). Now assume that the statement is true for $l$. Then

$$
\begin{aligned}
{\left[y_{i}, x_{j}^{l+1}\right]=} & {\left[y_{i}, x_{j}^{l}\right] x_{j}+x_{j}^{l}\left[y_{i}, x_{j}\right] } \\
= & u\left(\frac{x_{j}^{l}-x_{i}^{l}}{x_{j}-x_{i}}+\frac{x_{j}^{l}-\left(-x_{i}\right)^{l}}{x_{j}+x_{i}} c_{i} c_{j}\right)\left(1-e_{i} e_{j}\right) s_{i j} x_{j} \\
& -u\left(\frac{x_{j}^{l}-\left(-x_{i}\right)^{l}}{x_{j}+x_{i}}-\frac{x_{j}^{l}-x_{i}^{l}}{x_{j}-x_{i}} c_{i} c_{j}\right)\left(1+e_{i} e_{j}\right) \bar{s}_{i j} x_{j} \\
& +x_{j}^{l} u\left(\left(1+c_{i} c_{j}\right)\left(1+e_{j} e_{i}\right) s_{i j}-\left(1-c_{i} c_{j}\right)\left(1-e_{j} e_{i}\right) \bar{s}_{i j}\right) \\
= & u\left(\frac{x_{j}^{l+1}-x_{i}^{l+1}}{x_{j}-x_{i}}+\frac{x_{j}^{l+1}-\left(-x_{i}\right)^{l+1}}{x_{j}+x_{i}} c_{i} c_{j}\right)\left(1-e_{i} e_{j}\right) s_{i j} \\
& -u\left(\frac{x_{j}^{l+1}-\left(-x_{i}\right)^{l+1}}{x_{j}+x_{i}}-\frac{x_{j}^{l+1}-x_{i}^{l+1}}{x_{j}-x_{i}} c_{i} c_{j}\right)\left(1+e_{i} e_{j}\right) \bar{s}_{i j}, \\
{\left[y_{i}, x_{i}^{l+1}\right]=} & {\left[y_{i}, x_{i}^{l}\right] x_{i}+x_{i}^{l}\left[y_{i}, x_{i}\right] } \\
= & t c_{i} e_{i} \frac{x_{i}^{l}-\left(x_{i}^{l}\right)^{\tau_{i}}}{2}-v \frac{x_{i}^{l}-\left(x_{i}^{l}\right)^{\tau_{i}}}{2} \tau_{i}
\end{aligned}
$$




$$
\begin{aligned}
& -u \sum_{k \neq i}\left(\frac{x_{i}^{l}-x_{k}^{l}}{x_{i}-x_{k}}+\frac{x_{i}^{l}-\left(-x_{k}\right)^{l}}{x_{i}+x_{k}} c_{k} c_{i}\right)\left(1+e_{k} e_{i}\right) s_{k i} x_{i} \\
& -u \sum_{k \neq i}\left(\frac{x_{i}^{l}-\left(-x_{k}\right)^{l}}{x_{i}+x_{k}}-\frac{x_{i}^{l}-x_{k}^{l}}{x_{i}-x_{k}} c_{k} c_{i}\right)\left(1-e_{k} e_{i}\right) \bar{s}_{k i} x_{i} \\
& -u x_{i}^{l} \sum_{k \neq i}\left(\left(1+c_{k} c_{i}\right)\left(1+e_{k} e_{i}\right) s_{k i}+\left(1-c_{k} c_{i}\right)\left(1-e_{k} e_{i}\right) \bar{s}_{k i}\right)+t x_{i}^{l} c_{i} e_{i}-v x_{i}^{l} \tau_{i} \\
= & t c_{i} e_{i} \frac{x_{i}^{l+1}-\left(x_{i}^{l+1}\right)^{\tau_{i}}}{2 x_{i}}-v \frac{x_{i}^{l+1}-\left(x_{i}^{l+1}\right)^{\tau_{i}}}{2 x_{i}} \tau_{i} \\
& -u \sum_{k \neq i}\left(\frac{x_{i}^{l+1}-x_{k}^{l+1}}{x_{i}-x_{k}}+\frac{x_{i}^{l+1}-\left(-x_{k}\right)^{l+1}}{x_{i}+x_{k}} c_{k} c_{i}\right)\left(1+e_{k} e_{i}\right) s_{k i} \\
& -u \sum_{k \neq i}\left(\frac{x_{i}^{l+1}-\left(-x_{k}\right)^{l+1}}{x_{i}+x_{k}}-\frac{x_{i}^{l+1}-x_{k}^{l+1}}{x_{i}-x_{k}} c_{k} c_{i}\right)\left(1-e_{k} e_{i}\right) \bar{s}_{k i} .
\end{aligned}
$$

This completes the proof.

\section{A.1.5 Proof of Lemma 3.14}

It suffices to check the formula for every monomial $f$. First, we consider the monomial $g=$ $\prod_{j \neq i} x_{j}^{a_{j}}$. By induction and Lemma 3.13, we can show that the formula holds for the monomial of the form $g=\prod_{j \neq i} x_{j}^{a_{j}}$ (the detail of the induction step does not differ much from the following calculation). Now consider the monomial $f=x_{i}^{l} g$.

$$
\begin{aligned}
{\left[y_{i}, f\right]=} & {\left[y_{i}, x_{i}^{l}\right] g+x_{i}^{l}\left[y_{i}, g\right] } \\
= & t c_{i} e_{i} \frac{x_{i}^{l}-\left(-x_{i}\right)^{l}}{2 x_{i}} g-v \frac{x_{i}^{l}-\left(-x_{i}\right)^{l}}{2 x_{i}} \tau_{i} g \\
& -u \sum_{k \neq i}\left(\frac{x_{i}^{l}-x_{k}^{l}}{x_{i}-x_{k}}+\frac{x_{i}^{l}-\left(-x_{k}\right)^{l}}{x_{i}+x_{k}} c_{k} c_{i}\right)\left(1+e_{k} e_{i}\right) s_{k i} g \\
& -u \sum_{k \neq i}\left(\frac{x_{i}^{l}-\left(-x_{k}\right)^{l}}{x_{i}+x_{k}}-\frac{x_{i}^{l}-x_{k}^{l}}{x_{i}-x_{k}} c_{k} c_{i}\right)\left(1-e_{k} e_{i}\right) \bar{s}_{k i} g \\
& +t x_{i}^{l} c_{i} e_{i} \frac{g-g^{\tau_{i}}}{2 x_{i}}-v x_{i}^{l} \frac{g-g^{\tau_{i}}}{2 x_{i}} \tau_{i} \\
& -u \sum_{k \neq i} x_{i}^{l}\left(\frac{g-g^{s_{k i}}}{x_{i}-x_{k}}+\frac{g-g^{\bar{s}_{k i}}}{x_{i}+x_{k}} c_{k} c_{i}\right)\left(1+e_{k} e_{i}\right) s_{k i} \\
& -u \sum_{k \neq i} x_{i}^{l}\left(\frac{g-g^{s_{k i}}}{x_{i}+x_{k}}-\frac{g-g^{s_{k i}}}{x_{i}-x_{k}} c_{k} c_{i}\right)\left(1-e_{k} e_{i}\right) \bar{s}_{k i} \\
= & t c_{i} e_{i} \frac{f-f^{\tau_{i}}}{2 x_{i}}-v \frac{f-f^{\tau_{i}}}{2 x_{i}} \tau_{i}-u \sum_{k \neq i}\left(\frac{f-f^{s_{k i}}}{x_{i}-x_{k}}+\frac{f-f^{\bar{s}_{k i}}}{x_{i}+x_{k}} c_{k} c_{i}\right)\left(1+e_{k} e_{i}\right) s_{k i} \\
& -u \sum_{k \neq i}\left(\frac{f-f_{\bar{s}_{k i}}}{x_{i}+x_{k}}-\frac{f-f^{s_{k i}}}{x_{i}-x_{k}} c_{k} c_{i}\right)\left(1-e_{k} e_{i}\right) \bar{s}_{k i} .
\end{aligned}
$$

So the lemma is proved. 


\section{A.2 Proofs of Lemmas in Section 5}

\section{A.2.1 Proof of Lemma 5.11}

We will proceed by induction on $l$. For $l=1$, then the equations hold by the definition of $\mathbb{H}_{W}$. Now assume that the statement is true for $l$. Then

$$
\begin{aligned}
{\left[\eta_{i}, \xi_{j}^{l+1}\right]_{+}=} & {\left[\eta_{i}, \xi_{j}^{l}\right]_{+} \xi_{j}+(-1)^{l} \xi_{j}^{l}\left[\eta_{i}, \xi_{j}\right]_{+} } \\
= & u \frac{1}{\xi_{i}^{2}-\xi_{j}^{2}}\left(\xi_{i}^{l+1}-\xi_{j} \xi_{i}^{l}-\xi_{i} \xi_{j}^{l}+(-1)^{l} \xi_{j}^{l+1}\right)\left(s_{i j}+\bar{s}_{i j}\right) \xi_{j} \\
& +u \frac{\left(-\xi_{j}\right)^{l}}{\xi_{i}^{2}-\xi_{j}^{2}}\left(\xi_{i}^{2}-\xi_{j}^{2}\right)\left(s_{i j}+\bar{s}_{i j}\right) \\
= & u \frac{1}{\xi_{i}^{2}-\xi_{j}^{2}}\left(\xi_{i}^{l+2}-\xi_{j} \xi_{i}^{l+1}-\xi_{i} \xi_{j}^{l+1}+(-1)^{l+1} \xi_{j}^{l+2}\right)\left(s_{i j}+\bar{s}_{i j}\right), \\
{\left[\eta_{i}, \xi_{i}^{l+1}\right]_{+}=} & {\left[\eta_{i}, \xi_{i}^{l}\right]_{+} \xi_{i}+(-1)^{l} \xi_{i}^{l}\left[\eta_{i}, \xi_{i}\right]_{+} } \\
= & t \frac{\xi_{i}^{l}-\left(\xi_{i}^{l}\right)^{\tau_{i}}}{2 \xi_{i}} \xi_{i}+v \frac{\xi_{i}^{l}-\left(\xi_{i}^{l}\right)^{\tau_{i}}}{2 \xi_{i}} \tau_{i} \xi_{i} \\
& +u \sum_{k \neq i} \frac{1}{\xi_{i}^{2}-\xi_{k}^{2}}\left(\xi_{i} \xi_{k}^{l}-\xi_{k}^{l+1}-(-1)^{l} \xi_{i}^{l+1}+\xi_{k} \xi_{i}^{l}\right)\left(s_{i k}+\bar{s}_{i k}\right) \xi_{i} \\
& +t\left(-\xi_{i}\right)^{l}+v\left(-\xi_{i}\right)^{l} \tau_{i}+u \sum_{k \neq i} \frac{\left(-\xi_{i}\right)^{l}}{\xi_{i}^{2}-\xi_{k}^{2}}\left(\xi_{i}^{2}-\xi_{k}^{2}\right)\left(s_{k i}+\bar{s}_{i j}\right) \\
= & t \frac{\xi_{i}^{l+1}-\left(\xi_{i}^{l+1}\right)^{\tau_{i}}}{2 \xi_{i}}+v \frac{\xi_{i}^{l+1}-\left(\xi_{i}^{l+1}\right)^{\tau_{i}}}{2 \xi_{i}} \tau_{i} \\
& +u \sum_{k \neq i} \frac{1}{\xi_{i}^{2}-\xi_{k}^{2}}\left(\xi_{i} \xi_{k}^{l+1}-\xi_{k}^{l+2}-(-1)^{l+1} \xi_{i}^{l+2}+\xi_{k} \xi_{i}^{l+1}\right)\left(s_{i k}+\bar{s}_{i k}\right) .
\end{aligned}
$$

This completes the proof.

\section{A.2.2 Proof of Lemma 5.12}

It suffices to check the formula for every monomial $f$. First, we consider the monomial $g=$ $\prod_{j \neq i} \xi_{j}^{a_{j}}$. By induction and Lemma 5.11, we can show that the formula holds for the monomial of the form $g=\prod_{j \neq i} \xi_{j}^{a_{j}}$ (the detail of the induction step does not differ much from the following calculation). Now consider the monomial $f=\xi_{i}^{l} g$.

$$
\begin{aligned}
{\left[\eta_{i}, f\right]_{+}=} & {\left[\eta_{i}, \xi_{i}^{l}\right]_{+} g+(-1)^{l} \xi_{i}^{l}\left[\eta_{i}, g\right]_{+} } \\
= & t \frac{\xi_{i}^{l} g-\left(\xi_{i}^{l} g\right)^{\tau_{i}}}{2 \xi_{i}}+v \frac{\xi_{i}^{l} g-\left(\xi_{i}^{l} g\right)^{\tau_{i}}}{2 \xi_{i}} \tau_{i} \\
& +u \sum_{k \neq i} \frac{1}{\xi_{i}^{2}-\xi_{k}^{2}}\left(\xi_{i} \xi_{k}^{l}-\xi_{k}^{l+1}-(-1)^{l} \xi_{i}^{l+1}+\xi_{k} \xi_{i}^{l}\right)\left(s_{i k}+\bar{s}_{i k}\right) g \\
& +u \sum_{k \neq i} \frac{\left(-\xi_{i}\right)^{l}}{\xi_{i}^{2}-\xi_{k}^{2}}\left(\left(\xi_{i}-\xi_{k}\right) g^{s_{i k}}-\left(\xi_{i} g^{\tau_{i}}-\xi_{k} g^{\tau_{k}}\right)\right)\left(s_{i k}+\bar{s}_{i k}\right) \\
= & t \frac{\xi_{i}^{l} g-\left(\xi_{i}^{l} g\right)^{\tau_{i}}}{2 \xi_{i}}+v \frac{\xi_{i}^{l} g-\left(\xi_{i}^{l} g\right)^{\tau_{i}}}{2 \xi_{i}} \tau_{i} \\
& +u \sum_{k \neq i} \frac{1}{\xi_{i}^{2}-\xi_{k}^{2}}\left(\left(\xi_{i}-\xi_{k}\right)\left(\xi_{i}^{l} g\right)^{s_{i k}}-\left(\xi_{i}\left(\xi_{i}^{l}\right)^{\tau_{i}}-\xi_{k}\left(\xi_{i}^{l}\right)^{\tau_{k}}\right) g^{s_{i k}}\right)\left(s_{i k}+\bar{s}_{i k}\right)
\end{aligned}
$$




$$
\begin{aligned}
& +u \sum_{k \neq i} \frac{\left(-\xi_{i}\right)^{l}}{\xi_{i}^{2}-\xi_{k}^{2}}\left(\left(\xi_{i}-\xi_{k}\right) g^{s_{i k}}-\left(\xi_{i} g^{\tau_{i}}-\xi_{k} g^{\tau_{k}}\right)\right)\left(s_{i k}+\bar{s}_{i k}\right) \\
= & t \frac{\xi_{i}^{l} g-\left(\xi_{i}^{l} g\right)^{\tau_{i}}}{2 \xi_{i}}+v \frac{\xi_{i}^{l} g-\left(\xi_{i}^{l} g\right)^{\tau_{i}}}{2 \xi_{i}} \tau_{i} \\
& +u \sum_{k \neq i} \frac{1}{\xi_{i}^{2}-\xi_{k}^{2}}\left(\left(\xi_{i}-\xi_{k}\right)\left(\xi_{i}^{l} g\right)^{s_{i k}}-\left(\xi_{i}\left(\xi_{i}^{l} g\right)^{\tau_{i}}-\xi_{k}\left(\xi_{i}^{l} g\right)^{\tau_{k}}\right)\right)\left(s_{i k}+\bar{s}_{i k}\right) .
\end{aligned}
$$

So the lemma is proved.

\section{Acknowledgements}

This research is partially supported by NSF grant DMS-0800280. The main results of this paper for type $A$ were obtained at MSRI in 2006.

\section{References}

[1] Bazlov Y., Berenstein A., Noncommutative Dunkl operators and braided Cherednik algebras, arXiv:0806.0867.

[2] Drinfeld V., Degenerate affine Hecke algebras and Yangians, Funct. Anal. Appl. 20 (1986), 58-60.

[3] Dunkl C.F., Differential-difference operators associated to reflection groups, Trans. Amer. Math. Soc. 311 (1989), 167-183.

[4] Dunkl C.F., Opdam E.M., Dunkl operators for complex reflection groups, Proc. London Math. Soc. (3) 86 (2003), 70-108, math.RT/0108185.

[5] Etingof P., Ginzburg V., Symplectic reflection algebras, Calogero-Moser space, and deformed HarishChandra homomorphism, Invent. Math. 147 (2002), 243-348, math.AG/0011114.

[6] Ihara S., Yokonuma T., On the second cohomology groups (Schur-multipliers) of finite reflection groups, J. Fac. Sci. Univ. Tokyo Sect. I 11 (1965), 155-171.

[7] Karpilovsky G., The Schur multiplier, London Mathematical Society Monographs, New Series, Vol. 2, The Clarendon Press, Oxford University Press, New York, 1987.

[8] Khongsap T., Hecke-Clifford algebras and spin Hecke algebras III: the trigonometric type, arXiv:0808.2951.

[9] Khongsap T., Wang W., Hecke-Clifford algebras and spin Hecke algebras I: the classical affine type, Transform. Groups 13 (2008), 389-412, arXiv:0704.0201.

[10] Khongsap T., Wang W., Hecke-Clifford algebras and spin Hecke algebras II: the rational double affine type, Pacific J. Math. 238 (2008), 73-103, arXiv:0710.5877.

[11] Lusztig G., Affine Hecke algebras and their graded version, J. Amer. Math. Soc. 2 (1989), 599-635.

[12] Morris A., Projective representations of reflection groups, Proc. London Math. Soc. (3) 32 (1976), 403-420.

[13] Nazarov M., Young's symmetrizers for projective representations of the symmetric group, Adv. Math. 127 (1997), 190-257.

[14] Rouquier R., Representations of rational Cherednik algebras, in Infinite-Dimensional Aspects of Representation Theory and Applications (Charlottesville, 2004), Contemp. Math. 392 (2005), 103-131, math.RT/0504600.

[15] Schur I., Über die Darstellung der symmetrischen und der alternierenden Gruppe durch gebrochene lineare Substitutionen, J. Reine Angew. Math. 139 (1911), 155-250.

[16] Wang W., Double affine Hecke algebras for the spin symmetric group, math.RT/0608074.

[17] Wang W., Spin Hecke algebras of finite and affine types, Adv. Math. 212 (2007), 723-748, math.RT/0611950. 